\title{
Die Website des gesundheitstouristischen Betriebs: Vorschläge zur didaktischen Vermittlung ihres Registers für den Berufssprachenunterricht Deutsch im Tourismusbereich
}

https://dx.doi.org/10.12795/futhark.2014.19.06

Juan José Hernández Medina

Universidad de Almería

\begin{abstract}
The central pretense of this paper is to demonstrate the implementation of the Theory of the Register in the didactic preparation of an authentic commercial text Die Website des gesundheitstouristischen Betriebs in German classes for the purposes of tourism. The use of this textual demonstration in such classes responds to the linguistic needs of students of Tourism, Nursing and Physiotherapy who, among their possible occupational tasks, contemplate the possibility of working in the field of Health Tourism in Germany or in ressorts dedicated to this field with an ample German-speaking clientele. The text contains, indeed, numerous useful linguistic components for resolving key communicative situations in the mentioned field like, among others, the implementation of sanitary services or wellness or the task of marketing these.
\end{abstract}

The Theory of the Register becomes especially relevant to our teaching assignments, since it provides us with a series of parameters of analysis that are considerably effective for designing lessons about textual manifestations pertaining to specialized fields, such as the one that we discuss in the present investigation. On the one hand, these parameters have allowed us to identify the most frequent linguistic structures as well as the fundamental communicative context of a body of twelve websites pertain- 
ing to Health and Wellness Tourism companies. On the other hand, such parameters have served as a source of inspiration for the development of activities of contextual pragmatic nature intended for the assimilation of the aforementioned contextual linguistic contents as well as for the practice of learning strategies for the reception and production of a foreign language like in our case, German.

Keywords: Languages for Specific Purposes, Theorie of the Register, Socio-Pragmatic, Health Tourism

Abstract: In dem vorliegenden Artikel soll die Anwendung der Theorie des Registers bei der didaktischen Aufbereitung einer Textsorte aus dem Werbebusiness - dic Website des gesundleitstouristischen Betriebs - für den berufs- und fachbezogenen Fremdsprachenunterricht Deutsch in der Tourismusbranche dargestellt werden. Die Verwendung dieser textuellen Einheit in dem angesprochenen Fremdsprachenunterricht geht auf die Sprachbedürfnisse der Studenten der Studiengänge Tourismus, Gesundheits- und Krankenpflege sowie Physiotherapie zurück, welche die Möglichkeit in Betracht ziehen, in Deutschland in der Gesundheitstourismusbranche tätig zu werden und dadurch mit einer beträchtlichen Anzahl an deutschsprachiger Kundschaft in Berührung kommen werden. Der Text enthält zahlreiche lexikalische Einheiten und Sprachstrukturen, die zum Meistern von typischen deutschsprachigen Kommunikationssituationen in der angesprochenen Tourismusbranche nützlich sein können, so zum Beispiel für die Ausübung von Gesundheits- und Wellnessdienstleistungen oder die Erledigung von deren Marketings- oder Verkaufsarbeiten.

Der Theorie des Registers wird eine besondere Relevanz in unserer Lehrtätigkeit beigemessen. Sie verschafft uns eine Reihe von Analyseparametern, die von hoher Bedeutung für das Design von Themen zu Textsorten aus verschiedenen Fachbereichen sind. Einerseits haben uns diese Parameter ermöglicht die grundlegenden Sprach- und Situationskontextmerkmale eines aus zwölf Dienstleistungsbetriebswebseiten der Wellness- und Gesundheitstourismusbranche bestehenden Korpus zu identifizieren. Andererseits haben uns solche Parameter als Grundlage für die Ausarbeitung von Aufgaben pragmatisch-kontextueller Natur gedient. Diese sind notwendig zur Vermittlung jener grundlegenden sprach- und kontextbezogenen Merkmale und zur Einübung einer beträchtlichen Anzahl von rezeptiven und produktiven Lernstrategien beim Erwerb 
einer Fremdsprache, wie im vorliegenden Fall bei dem Erwerb der deutschen Sprache.

Schlüsselwörter: Berufs- und Fachsprachenunterricht, Theorie des Registers, Soziopragmatik, Gesundheitstourismus.

\section{EINLEITENDE BEMERKUNGEN}

Der deutschen Sprache kommt in der touristischen Gesundheitsund Wellnessbranche Europas ein hoher Wert zu. Tatsächlich gilt Deutschland als Wegbereiterland in diesem Tourismussektor. Schon im XIX. Jahrhundert befand sich dort die berühmteste Hydroklinik des europäischen Kontinents. Diese hatte ihren Standort in BadenBaden, ein Kurort, der für seine heilenden Gewässer bekannt ist und mitten im Herzen des Schwarzwaldes liegt. Dabei handelte es sich um einen auf die Vorsorge und Behandlungen von Leiden, wie unter anderen Asthma oder Rheuma, spezialisierten touristischen Gesundheitskomplex (Vogeler Ruiz/ Hernández Armand, 1995: 273).

Der besagte Tourismusbereich stellt eine bedeutende Einkommensquelle für die deutsche Wirtschaft dar. Mehr als ein Drittel der rund 407 Millionen gebuchten Übernachtungen im Tourismusbereich im Jahr 2012 in Deutschland fanden in Kur- und Badeorten statt (Ökonomisches Schwergewicht: Wir sind Gesundheitstourismus!). Die von den Gesundheits- und Wellnesstouristen aus aller Welt produzierten Einnahmen in diesem Land belaufen sich jährlich auf ungefähr 930 Millionen Euro (Juszczak, 2012: 3).

Andererseits genießt der Gesundheits- und Wellnesstourismus eine beträchtliche soziale Anerkennung unter den Deutschen und, wie allgemein bekannt ist, verfügen diese über eine hohe Kaufkraft. $36 \%$ von ihnen halten es für sehr wichtig, während ihres Urlaubs „etwas für die Gesundheit zu tun" (Innovativer Gesundheitstourismus in Deutschland, 2011). Ein Großteil der deutschen Reisenden misst den Gesundheits- und Wellnessprodukten einen Großen Wert zu (Plan General de Turismo Sostenible de Andalucia 2008 - 2011, 84; und Bosch, 1998: 68). Die schon erwähnte hohe Kaufkraft erzeugt eine immer 
gröfer werdende Nachfrage nach diesen Produkten und wird während der Urlaubsperiode oder Freizeit sowohl im eigenen Land als auch auferhalb der Grenzen eingesetzt.

Auf die angesprochene potenzielle Nachfrage sind zahlreiche nationale und internationale auf Gesundheitsdienstleistungen spezialisierte Tourismusuntemehmen aufmerksam geworden. Um deutsche Touristen anzulocken und somit ihre Verkäufe zu potenzieren haben diese Dienstleistungsbetriebe eine Serie verschiedenster Maßnahmen unternommen, von denen einige im Folgenden aufgezählt werden: Umsetzung von gemeinsamen Arbeitsstrategien zwischen deutschen Touroperatoren und Touroperatoren des Reiseziellandes, Durchführung von Forschungen zu den von deutschsprachigen Touristen am meisten nachgefragten Wellness- und Gesundheitsdienstleistungen sowie Ausarbeitung neuer Marketing- und Kommerzialisierungsstrategien dieser Produkte. Eine der unentbehrlichsten und wirkungsvollsten Marketing- und Kommerzialisierungsstrategien besteht übrigens in der Erstellung der in dieser Arbeit behandelten Textsorte: die Website des gesundheitstouristischen Betriebs.

Die vorliegende Arbeit hat die Sprachbedürfnisse der Studenten der Studiengänge Touristik, Gesundheits- und Krankenpflege und Physiotherapie als grundlegende Motivation, welche die Möglichkeit in Betracht ziehen, in der wellness- und gesundheitstouristischen Branche Deutschlands oder in auf diesen Bereich spezialisierten Tourismusunternehmen mit einem großen Anteil deutschsprachiger Kundschaft $\mathbf{z u}$ arbeiten. Im Rahmen dieses Beitrags werden praktische Arbeitsvorschläge zur didaktischen Aufbereitung der sprach- und kontextuellen Niveaus der Website des gesundheitstouristischen Betriebs vorgestellt. Die besagte didaktische Aufbereitung ist durch die Implementierung der Theorie des Registers und durch Sprachlernstrategien gelungen.

Die Meinungen unserer aktuellen und ehemaligen Deutschlernenden sowie unsere Untersuchungen zu der Website des gesundheitstouristischen Betriebs lassen uns schließen, dass diese Textsorte aus der Werbebranche nützliche lexikalischen Einheiten und Sprachstrukturen zur Meisterung typischer 
Kommunikationssituationen im Wirtschaftszweig Gesundheitstourismus enthält, wie unter anderem, die Erledigung von Kommerzialisierungsarbeiten und die Ausübung von Wellnessdienstleistungen.

Darüber hinaus geht es bei der Website des gesundheitstouristischen Betriebs um einen Schlüsseltext im deutschsprachigen gesundheitstouristischen Wirtschaftszweig. Durch diese werbungsgeschäftliche Textsorte versucht der Gesundheits- oder Wellnessdienstleistungsbetrieb zu erreichen, dass sein Angebot an Produkten und Dienstleistungen bei einem möglichst großen Publikum ankommt. Eine solche Website enthält nämlich attraktive Beschreibungen über die zu bewerbende Sache und ermöglicht deren Erwerb jederzeit per einfachem Mausklick.

Der Theorie des Registers wird eine besondere Relevanz in unseren Lehrtätigkeiten beigemessen. Sie verschafft uns nämlich eine Serie von Analyseparametern von beträchtlicher Wirksamkeit für das Design von Themen zu Textsorten aus verschiedenen Fachbereichen, wie z.B. das in diesem Artikel behandelte Thema. Einerseits haben uns diese Parameter ermöglicht die grundlegenden Sprach- und Situationskontextmerkmale der Website des gesundheitstouristischen Betriebs zu identifizieren. Andererseits dienen uns solche Parameter als Grundlage für die Ausarbeitung von Aufgaben pragmatischkontextueller Natur, zur Vermittlung jener grundlegenden sprachund kontextbezogenen Merkmale, sowie zur Einübung einer beträchtlichen Anzahl von rezeptiven und produktiven Sprachlernstrategien.

Den Strategien zum Erwerb von Fremdsprachen schreiben wir ebenfalls eine bedeutende Rolle in unserem Unterrichtskonzept zu. Wie es allgemein bekannt ist, vereinfachen und optimieren sie den Lernprozess der Zielsprache. In unserem Fach- und Berufssprachenunterricht „Deutsch im Tourismusbereich" werden die erwähnten Strategien in der Regel zur Aneignung der sprach- und situationskontextbezogenen Niveaus von authentischen tourismusbezogenen Geschäftstexten wie z. B. die Website des gesundheitstouristischen Betriebs eingesetzt. 
Der folgende Teil des vorliegenden wissenschaftlichen Beitrags gliedert sich in fünf Abschnitte. Zunächst wird eine kurze Zusammenfassung über die relevantesten allgemeinen Merkmale des Gesundheitstourismus dargestellt. Danach wird die Theorie des Registers präsentiert. Der dritte Abschnitt handelt von Strategien für den Erwerb von Fremdsprachen. Die Ergebnisse der Applikation verschiedener Analyseparameter dieser Theorie auf ein Korpus von Websites eines gesundheitstouristischen Betriebs sind Inhalt des vierten Abschnitts: die Analysephase. Der letzte Abschnitt konzentriert sich auf die Gestaltung einer Unterrichtseinheit, in der die Aufgaben geschildert werden, die auf der Basis dieser Ergebnisse und der Theorie des Registers ausgearbeitet worden sind. Abschließend werden die gewonnenen Erkenntnisse über die positiven Aspekte der Implementierung der Theorie des Registers bei der didaktischen Aufbereitung der Ausgangstexteinheit vorgestellt.

\section{DER GESUNDHEITSTOURISMUS: ALLGEMEINE ASPEKTE}

Die Motive, aus denen sich Menschen entscheiden, zu verreisen, sind zahlreich. Fachleuten aus dem Tourismusbereich zufolge ist eines der Motive der Gesundheitstourismus; eine der ältesten Tourismusmodalitäten der Welt. Diese Tourismusmodalität basiert auf der therapeutischen Nutzung der Thermalquellen, die schon in der hellenistischen Welt im 5. Jahrhundert vor Christus stattfand und die zu einem Sozialphänomen bedeutsamer Relevanz in der römischen Welt wurde. Eine bedeutsame Anzahl großer Städte dieser Zivilisation verfügte über Kurorte und Heilbädereinrichtungen mit medizinischen Betreuungsdienstleistungen, in denen auch soziale und kulturelle Aktivitäten organisiert wurden.

Zeugnisse dieser großen historischen Vergangenheit des Gesundheitstourismus finden wir auf dem gesamten europäischen Kontinent an verschiedenen Orten, deren Namen unter anderem Termini wie "Bad", "Wasser" oder "Thermen" beinhalten, wie beispielsweise "Bad" in Bad Neunahr und Bad Godesberg (Deutschland), "Calde" in Caldes de Montbui und Caldes d'Estrac 
(Spanien), "Bain" in Divonne-er-Bains und Dign-Les-Bains (Frankreich), "Lazne" in Marianske Lazne und Frantiskovy Lazne (Tschechien), "Terme" in "Acqui Terme" und "Abano Terme" (Italien), usw. Bei diesen Städten handelt es sich um solche, die einen beträchtlichen wirtschaftlichen Aufschwung während des letzten Drittels des neunzehnten Jahrhunderts erlebten, der Glanzepoche des Wassertourismus.

Der Gesundheitstourismus stellt große Wirtschaftsaussichten in der Europäischen Gemeinschaft dar. Seit dem letzten Jahrzehnt hat er von zahlreichen Länder- und EU-Werbemaßnahmen profitiert. Diese Maßnahmen bestanden grundsätzlich in Plänen zu seiner Reaktivierung, Modernisierung und strukturellen Neuorientierung. Auf europäischer Ebene hebt sich das Projekt "Thermae Europae" hervor. Dieses Projekt wurde zwischen den Jahren 2000 und 2009 von zwölf europäischen Städten mit einer reichen Tradition im Bereich des thermalen Wassertourismus entwickelt und durchgeführt: Spa (Belgien), Vichy (Frankreich), Acqui Terme (Italien), Salsomaggiore (Italien), Bagni di Lucca (Italien), Chaves (Portugal), Frantiskovy (Tschechien), Lázně Karlovy Vary (Tschechien), Mariánské Lázně (Tschechien), Bath (Vereinigtes Königreich) und Piešt'any (Slowakei). Dieses Projekt hatte die Förderung des Wassertourismus in Europa zum Ziel. Zu diesem Zweck wurden dabei unter anderem folgende Hauptvorgehenspläne entwickelt und durchgeführt: Verstärkung der Zusammenarbeit, Austausch von "Know-how"-Ideen und Erfahrungen, Herausgabe von gemeinsamen Fachveröffentlichungen, fachlicher Beistand und die Veranstaltung von Seminaren sowie umfassende Marktforschungen zum betreffenden Thema (European Route of thermal heritage and thermal towns, Acqui Terme: European Historical Thermal Town Association, 8).

Unter den von EU-Mitgliedsstaaten ausgeführten Plänen zur Förderung des Gesundheitstourismus ragen die von Österreich und Spanien unternommenen Pläne heraus. In Österreich wurde während des Jahres 2002 das Projekt „Wellbeing Destination Austria GmbH" in Gang gesetzt. Ziel dieses auf staatliche und regionale Initiativen zurückgehenden Programms war es, dessen Gesundheitstourismusdienstleistungsbereich zur Markenware mit 
bewährter Qualităt zu verhelfen. Es wurde versucht, dieses Vorhaben mittels der Entwicklung von innovativen Dienstleistungen und Produkten für den internationalen Markt zu erreichen, da das Alpenland beabsichtigte, sich langfristig zu einem europäischen gesundheitstouristischen Vorreiterzielland zu verwandeln (Bässler, 2003: 8).

Ihrerseits rief die spanische Verwaltung „das Programm zur Unterstützung der Spanischen Föderation von Gemeinden und Provinzen bei der Entwicklung von Aktivitäten zur Förderung des Wassertourismus" 2006 ins Leben (Real Decreto 1143/2006). Dieses Programm hatte unter anderem zum Ziel den guten Ruf Spaniens als Urlaubszielland auszunutzen, um diese Tourismusmodalität wieder in Schwung zu bringen; eine größere Implikation der Gemeinden in der Nutzung ihrer eigenen Thermalwasserressourcen zu erreichen, die entsprechenden gesundheitstouristischen Infrastrukturen $\mathrm{zu}$ renovieren und das adäquateste Tourismusangebot für seine Projizierung auf nationale und internationale Ebene auszuarbeiten (Ibid.).

Die Initiativen des Privatbereichs haben sich als ein Schlüsselfaktor zur Überwindung der Krise in der gesundheitstouristischen Branche entpuppt. Unter ihnen sind die von Unternehmern von Thermalwasserbetrieben unternommenen Maßnahmen zur Erneuerung und Umstrukturierung ihrer Wasseranlagen zu erwähnen, welche als Hauptziel die Verbannung des traditionellen Images der Kurorte, gleichbedeutend mit Krankenhäusern, hatten, zu denen Patienten nur zur medizinischen Behandlung gingen. Kurz gefasst beabsichtigten die besagten Initiativen grundsätzlich auf der Basis des körperlichen und seelischen Gesundheitstourismus ein neues Dienstleistungsmodell für diese Wasseranlagen zu entwickeln. Auf diese Weise wurden zahlreiche Anlagen der angesprochenen Tourismusbranche mit den notwendigen Infrastrukturen für das Angebot der innovativen Gesundheits- und Wellnessdienstleistungen versehen, wie beispielsweise Fitness-, Beauty- und Kosmetikzentren.

Der Gesundheitstourismus hat ebenfalls eine heftige konzeptionelle Diskussion veranlasst. Zur Erleichterung der Lektüre der vorliegenden Arbeit beschränken wir uns auf nur eine, allerdings 
u.E. erhellende Definition über das Phänomen, die von Kaspar, einem Fachmann für die Welt der Reisen, in der man seine grundsätzliche Ansicht erkennen kann: „Die Gesamtheit der Beziehungen und Erscheinungen, die sich aus der Ortsveränderung und dem Aufenthalt von Personen zur Förderung, Stabilisierung und gegebenenfalls Wiederherstellung des körperlichen, geistigen und sozialen Wohlbefindens unter der Inanspruchnahme von Gesundheitsleistungen ergeben" (Kaspar zitiert in Bässler, 2003: 19).

Jene Tourismusmodalität lässt sich in zwei Hauptideen aufteilen: Der Medizintourismus und der Wellness- und Beautytourismus. Die erste Modalität bezieht sich grundsätzlich auf die Inanspruchnahme nach ärztlicher Behandlung seitens einer unter einer Krankheit oder Schmerzen leidenden Person. Ein Patient unternimmt zum Beispiel eine Reise in einen Kurort eines bestimmten Landes, um sich dort einer Therapie zu unterziehen'.

Der Wellness- und Beautytourismus hingegen wird größtenteils von Menschen ohne Bedarf an ärztlicher Behandlung ausgeübt. Neben der Instandhaltung und der Stärkung der Gesundheit hat diese Art des Tourismus als grundsätzliche Motivationen die neue Mode des Schönheitskults, die Knüpfung von sozialen Kontakten sowie die Flucht aus einer städtischen, stressigen und von Berufsverantwortungen verunreinigten Welt. Als Beispiel dazu lässt sich der Fall eines völlig gesunden Managers mit großer Betriebsverantwortung erwähnen, der einen Teil seines Urlaubs in einer schönen maritimen Gegend gelegenen Hotelanlage mit medizinischen Wellnessinfrastrukturen verbringt, wo er nicht nur die Möglichkeit hat, an Wohlstandsaktivitäten teilzunehmen, sondern auch neue soziale Kontakte zu knüpfen.

$\mathrm{Zu}$ ihrer Ausführung benötigen die gesundheitstouristischen Dienstleistungen Anlagen mit speziellen Infrastrukturen. In groben Zügen lässt sich eine vierteilige Einteilung dieser Anlagen ausmachen:

1 Die Chiropraktik, Knochentherapie, Physiotherapie, Dermatologie und Balneotherapie machen übrigens die relevantesten Gesundheitsbehandlungen des Medizintourismus aus. Ein bedeutsamer Teil der gesundheitstouristischen Produkte kombiniert mehrere von ihnen. 
Kurorte oder Thermalanlagen,

Thalassotherapiezentren und Dia-Spa-Anlagen.

Wellness-Hotels,

Die Kurorte oder Thermalanlagen bieten wassertherapeutische Dienstleistungen mit heilenden Wässem an, die von ärztlichen Fachkràften beaufsichtigt werden (Bässler, 2003: 10; López Morales, 2003: 125; und Plan General de Turismo sostenible de Andalucía 20082011, 118). Im Allgemeinen bestehen diese Gesundheitsdienstleistungen in individualisierten Behandlungen, die von Fachärzten für die zu behandelnden Leiden vorgeschrieben wurden (Bässler, 2003: 19; und Schroeder, 2002: 205).

Das ursprünglich auf thermale Wässer zurückgehende Gesundheitsangebot ist übrigens nicht das einzige Angebot, mit dem zahlreiche Kurorte oder Thermalanlagen aufwarten. Wie oben schon mehrfach bei der Darstellung der Initiativen der Unternehmer der Tourismusbranche zur Modernisierung sowie Erneuerung des Gesundheitstourismus erwähnt wurde, ist eine beträchtliche Anzahl dieser Anlagen mit zusätzlichen Infrastrukturen für die Leistung von Wellness- und Beautyleistungen ausgestattet, mittels derer sie ihr traditionelles Angebot ergänzen können. Auf diese Weise erweitern sie ihren Kundenbestand. Ihre Kundschaft besteht demzufolge nicht ausschließlich aus Klienten mit Bedarf an Krankenpflege.

Die Wellness-Hotels richten ihr Augenmerk besonders auf sogenannte Leistungsbündel, die aus einem oder mehreren Dienstleistungen bestehen. Dazu zählen unter anderem: Relax, Fitness, Ernährung, Beauty und Bäderservices (Bässler, 2003: 20-21). Die Kundenbetreuung in diesen Hotels wird in der Regel von Fachpersonal für Wellness oder von medizinischen Fachkräften geleistet. In vielen Fällen werden die Pflege der Patienten sowie die zuvor angeführten Leistungsbündel von Fachärzten beaufsichtigt. In diesem Fall kann diese Pflege medizinische Untersuchungen einschließen, durch Diäten ergänzt werden oder die Heilung von Schmerzen und Krankheiten zum Ziel haben.

Wie ihr eigener Name angibt, bieten die Thalassotherapiezentren therapeutische Dienstleistungen zur Schönheitspflege sowie zur Heilung von mentalen oder körperlichen Leiden an. In den meisten 
Fällen werden die angesprochenen Dienstleistungen unter medizinischer Beaufsichtigung realisiert. Die meisten dieser Gesundheitsanlagen ergänzen das besagte Angebot mit typischen Wellness-Hotel-Produkten.

Die Dia-Spas oder städtischen Kurorte legen den Akzent auf „Balneotherapie- und Hydrotherapiedienstleistungen”, die sie im "Allgemeinen mit anderen, nicht auf Wasser basierenden, gesundheitstourismusbezogenen Leistungen ergänzen oder kombinieren. Solche Therapien werden grundsätzlich bei der Schönheitspflege oder mentalen Betreuung angewandt, sind von kurzer Dauer (ein paar Stunden oder einen Tag) und richten sich nach Kunden aus, die nur einen kurzen Aufenthalt im jeweiligen Urlaubsort verbringen.

\section{DIE THEORIE DES REGISTERS}

Seit ihrer Entstehung ist die Theorie des Registers mit der Berufsund Fachsprachendidaktik eng verbunden gewesen: Der Berufs- und Fachsprachenunterricht Englisch (English for Specific Purposes). Wie man der Lektüre akademischer Werke über diesen Bereich entnehmen kann, ist die erste Phase dieser Berufs- und Fachsprachendidaktik unter der besagten Benennung bekannt Jordan, 1997: 228; Hutchinson/ Waters, 1987: 9; und García Mayo, 2000: 29). Diese Tatsache lässt sich aus ihrer Relevanz als didaktischer Ansatz in jenem Fremdsprachenunterrichtsbereich erklären.

Diese Theorie schreibt der linguistischen Kompetenz und der kommunikativen Kompetenz eine große Rolle bei der Programmierung des Berufs- und Fachsprachenunterrichts zu und beschäftigt sich mit Varietäten der Sprache, die auf dem Sprachgebrauch in einem bestimmten Situationskontext basieren. Ein bestimmter Sprecher wird je nach Situation - also Zeit, Umstand, Ort usw. - ein bestimmtes Register anwenden. Typische Register sind z. B. die Fachsprache im Tourismusbereich, die Wirtschaftssprache, die Fachsprache der Medizin usw. Der Lernende muss also nicht nur wissen, wie man Sätze in der Zielsprache richtig konstruiert, sondern auch die Konventionen des 
Sprachverhaltens und des Sprachgebrauchs beherrschen. Demzufolge sollten die von der Theorie des Registers ausgehenden Lehrprogramme für den Berufs- und Fachsprachenunterricht die häufigsten sprachlichen Repertoires der zu erlemenden Varietät der Zielsprache beinhalten. Einerseits wurden jene Varietäten sprachlichen Frequenzuntersuchungen zu der Identifizierung und Systematisierung ihrer am häufigsten lexiko-grammatikalischen auftretenden Eigenschaften unterzogen. Andererseits untersuchte man die Integration solcher Sprachrepertoires in das Unterrichtskonzept des jeweiligen berufs- oder fachbezogenen Fremdsprachenunterrichts. Wie Alcaraz Varó (2000: 23) feststellt, entstand jene theoretische Analyseperspektive "mit dem Ziel, eine Studien- und Systematisierungsmethodologie der verschiedenen Varietäten in einer Sprache gemäß dem von ihnen gemachten Gebrauch" zu erstellen.

Die theoretischen Grundsätze der Theorie des Registers gehen auf die von Firth während der fünfziger Jahre aufgestellten strukturalistischen sprachsystematisch-orientierten Postulate zurück. Bei diesen Postulaten findet man zwei Schlüsselkonzepte zur Entfaltung der besagten Theorie: "der Kontext der Sprechsituation" und die "Restricted languages". Der erste Begriff spielt auf ein Handlungsverhaltensschema an, nach dem sich der Teilnehmer an sozialen Kommunikationsvorgängen richtet und welches von einer Serie handlungsorientierter Variablen abhängt:
A. The participants: persons, personalities and relevant features of these:
1. The verbal action of the participants
2. The non-verbal action of the participants.
$B$. The relevant objects and non-verbal and non- personal events
C. The effect of the verbal action (Firth zitiert in Robins, 1997: 216 - 217).

Der Terminus "restricted languages“ weist evidente Analogien zu dem bekannten Konzept des "Registers" auf. Dieses spielt auf mit eigenen Grammatikregeln und Wortschatz versehene linguistische Varietäten an, welche in empirisch eingeschränkten 
Kommunikationsfeldern angewandt werden (Firth zitiert in De Beaugrande, 1993: 8; Rucci, 1999: 192; Suau Jiménez, 2001; und Zequan, 2003). Unter den beschränkten Sprachen sind die persönlichkeitsbezogenen Stile, die Sprachen der Wissenschaft, der Industrie, des Sports, der Politik, des Handels, der Technologie, der Meteorologie, etc. hervorzuheben (De Beaugrande, 1993: 8; Rucci, 1999: 192; und Zequan, 2003).

Die erste bedeutsame wissenschaftliche Monografie zur Theorie des Registers ist The Linguitic Sciences and Language Teaching (Halliday et al., 1964). Bei diesem Werk geht es um eine angewandte Linguistikabhandlung zur Didaktik des Englischen als Fremdsprache. Das Werk beinhaltet eine Serie von Beschreibungen, welche nach unserer Auffassung einen angemessenen Einblick in die theoretischkonzeptionelle Natur des Terminus Register verschafft. In jenem Essay wird nämlich die berühmte Definition dargestellt, in der dessen Abgrenzung zu dem Terminus des Dialekts expliziert wird. Der Dialekt wird als eine von der Situation, in der sich der Kommunikationsteilnehmer befindet, nicht bedingte linguistische Varietät beschrieben. Der Terminus Register bezieht sich dagegen auf eine von deren Gebrauch in einem bestimmten Situationskontext geprägten sprachlichen Varietät:

Some of the questions that can be asked about a language comunity and its language are these. First, what happens when it impinges on other language communities? Second, what varieties of its language are there? Under the second question come these subdivisions: varieties according to users (that is, varieties in the sense that each speaker uses one variety and uses it all the time) and varieties according to use (that is, in the sense that each speaker has a range of varieties and chooses between them at different times). The variety according to user is a DIALECT; the variety according to use is a register. Third, what attitudes do the speakers display towards their language and any or all of its varieties? (77).

A dialect is a variety of language distinguished according to the user: different groups of people whithin the language community speak different dialects. It is possible also to recognize varieties of a language along another dimension, ditinguished according to use. Language varies as its function varies, it differs in different situations. The name 
given to a variety of a language distinguished according to use is register (Id., 87).

In den nachfolgenden Ausführungen jenes Werkes wird der Zweck der Kategorie des Registers näher erläutert. Diese Kategorie ist notwendig, um den Gebrauch zu erklären, den die Gesprächspartner von ihrer Sprache machen:

The category of "register" is needed when we want to account for what people do with their language, for example when we observe language activity. It is only by reference to the various situations, and situation types, in which language is used that we can understand its funcioning and its effectivenes. Language is not realized in the abstract: it is realized as the activity of people in situations, as linguistic events which are manifested in a particular dialect and register (Halliday et al., 1964: 87).

Aus der Lektüre einiger Reflexionen in dem vierten Kapitel des angesprochenen Werkes lässt sich die Relevanz des Frequenzgrades der lexikal und grammatikalisch bezogenen Bestandteile in den methodischen Postulaten der Registertheorie ableiten: "But the crucials of any given register are to be found in its grammar and its lexis. Probably lexical features are the most obvious. Some lexical items suffice almost by themselves to identify a certain register" (Halliday et al., 1964: 87).

Später sind zahlreiche theoretische Essays zu der Theorie des Registers herausgegeben worden. Eine gemäß der im Linguistikbereich vorherrschenden Meinung herausragende Arbeit ist der Text Language as a Social Semiotic (Halliday, 1978). Sie hat als Eingebungsquelle für weitere zahlreiche Werke auf der Grundlage dieses lern- und lehrorientierten Prinzips gedient. Dabei handelt es sich um ein sprachsystematisch orientiertes und wissenschaftliches Handbuch über soziolinguistikbezogene Schwerpunktthemen, aus dem unserer Meinung nach interessante Erkenntnisse über ihre Applikationsmöglichkeiten auf den Berufs- und Fachsprachenunterricht entnommen werden können. In dem besagten Standardwerk werden Definitionen formuliert, die einen ausdrücklicheren Überblick über die theoretisch-konzeptionelle Natur der Theorie des Registers ermöglichen. In einer dieser 
Formulierungen wird der Zweck dieser Theorie festgelegt. Dabei geht es um die Aufdeckung der allgemeinen Prinzipien, nach denen sich die kontextuelle Variation der Sprache richtet, sowie um die die sprachlichen Merkmale bestimmenden Situationsfaktoren: "What the theory of register does is to attempt to uncover the general principles which govern this variation (language variation in context), so that we begin to understand what situational factors determine what linguistic features"(32).

In dem zweiten Abschnitt des zweiten Kapitels wird eine Definition von der Theorie des Registers formuliert, die einen neuen Parameter einschließt: den semantischen Parameter. In dieser Formulierung wird der Registerterminus als eine Vielfalt von Sprachund Bedeutungsressourcen kategorisiert, die durch sozio-kulturelle Faktoren und den spezifischen Grad des Situationskontextes determiniert werden:

A register can be defined as the configuration of semantic resources that the member of a culture typically associates with a situation type. It is the meaning potential that is accessible in a given social context. Both the situation and the register associated whith it can be described to varying degrees of specifity; but the existence of registers is a fact of everyday experience - speakers have no difficulty in recognizing the semantic options and combinations of options that are 'at risk' under particular enviromental conditions. Since these options are realized in the form of grammar and structures. But it is defined in terms of meanings; it is not an aggregate of conventional forms of expression superposed on some underlying content by 'social factors' of one kind or another. It is the selection of meanings that constitutes the variety to which a text belongs (Halliday, 1978: 111).

Auf die gleiche Weise werden bei dieser wissenschaftlichen Arbeit die Hauptparameter des Registeransatzes erläutert. Diese bestehen in drei kontextuell-pragmatischen Variablen: "das, was gerade geschieht", "das in Aktion auftretende sprachliche Handlungsfeld" und "der Kommunikationsteilnehmer". Diese legen durch Kombination die sprachliche Varietät fest, in der sowohl die Bedeutungsmerkmale als auch die für ihre Äußerung verwendeten Sprachformen ausgewählt werden: 
Types of linguistic situation differ from one another, broadly speaking, in three respects: first, as regards what actually is taking place; second$\mathrm{ly}$, as regards what part of the language is playing; and thirdly, as regards who is taking part. These three variables, taken together, determine the range within which meanings are selected and the forms which are used for their expression. In other words they determine the "register" (Halliday, 1978: 31).

Ebenso nimmt Halliday (1978) eine dreigliedrige Einteilung zur Theorie des Registers aus dem 1964 erschienenen Werk wieder auf. In dieser Arbeit werden drei Makrofunktionen der Sprache vorgelegt: das Feld ( $d$. h. besprochene Themen oder Angelegenheiten), der Tenor (d. h. der Formalitäts- oder fachsprachliche Grad) und der Modus (d. h. der schriftliche oder gesprochene Kanal). Diese Makrofunktionen wirken über alle Sprachniveaus (d. h. semantisches Niveau, phonologisches Niveau, lexikalisches Niveau und morphosyntaktisches Niveau) und bestimmen die semantischfunktionellen Beziehungen zwischen den sprachlichen Äußerungen und dem situationellen Verwendungszusammenhang systematisch. Sie beeinflussen das dabei verwendete Register. Hierzu halten wir es für angebracht zwei Reflexionen über die Theorie des Registers in Halliday's Ausführungen hervorzuheben, die unseres Erachtens an Kommunikationsstrategien zur Voraussage von mit gewisser Wahrscheinlichkeit in einer gewissen Kommunikationssituation in der Zielsprache produzierten sprachlichen Äußerungen erinnern (Glaboniat et al., 2002). Die erste dieser Überlegungen spielt auf die "Merkmale des Vorhersagens" („form of prediction") über die Sprache an, die in einem gewissen sozialen Verwendungszusammenhang verwendet werden kann: „The notion of register is thus a form of prediction: given that we know the situation, the social context of language use, we can predict a great deal about the language that will occur" (Halliday, 1978: 32). In der anderen Überlegung wird erwähnt, dass die Kenntniss der drei Makrofunktionen des Diskurses (das Feld, der Tenor und der Modus) die Vorhersage von für einen situationellen Kontext typische Spracheinheiten ermöglicht: „What we need to know about a context of situation in order to predict the linguistic features that are likely to be associated with it has been summarized under three headings: we need to 
know the 'Field of discourse', the 'tenor of discourse' and the 'mode of discourse" (Id. 33).

An der Theorie des Registers werden aber auch negative Kritiken geübt. So wird unter anderem kritisiert, eine übermäßige Konzentration auf den formellen und grammatischen Bereich zu richten (Hutchinson/ Waters 1987: 10-11; García Mayo, 2000: 32 - 33), sowie einen geringen Nutzen für die didaktische Vermittlung des pragmatischen Charakters einer Fachsprache zu bieten (García Mayo, 2000: 33). Wir jedoch halten solche Kritiken für ungerecht und unangebracht.

In dem uns betreffenden Fall, also dem berufs- und fachbezogenen Fremdsprachenunterricht Deutsch im Tourismusbereich, hat sich diese Methodologie als sehr nützlich erwiesen. Einerseits erlaubt sie uns einen wesentlichen Teil der für den Tourismus typischen Textsorten auf sprachliche und kontextuelle Prozesse hin zu untersuchen. Andererseits dienen uns die aus ihrer Applikation gewonnenen Erkenntnisse als Basis für die Schaffung von an den pragmatisch-funktionellen zielsprachlichen Kontext angepassten Lernaufgaben. Hierzu ist es auch angebracht anzuführen, dass die Theorie des Registers nicht das einzige in unserem forschungsdidaktischen Bereich verwendete Untersuchungsverfahren ist $^{2}$. Und falls man es vorzieht, formelle, situationskontext- oder grammatikübergreifende Untersuchungen zu realisieren, empfehlen wir, die Genreanalyse heranzuziehen. Dabei geht es um eine textbezogene Analyseart, die ebenfalls häufig im angewandten Linguistikbereich zur Beschreibung des möglichen Einsatzes von authentischen Texten auf den Fremdsprachenunterricht verwendet wird.

Beide analytischen Konzeptionen beziehen sich also auf verschiedene pragmatisch-funktionelle Handlungsfelder der Sprache. Wenn wir uns auf das sprachkontextuelle Niveau konzentrieren, werden wir also die Theorie des Registers heranziehen. Wenn wir uns andererseits mit dem satzübergreifenden textuellen Niveau

2 Interessant ist dabei auch die Behandlung der Tourismussprache aus Sicht der kognitiven Metapherntheorie. Vgl. dazu Corbacho Sánchez (2014).

Futhark 9 (2014)

Hernández, Die Website, 153-202

ISSN 1886-9300 
beschäftigen, werden wir auf die Genreanalyse zurückgreifen. Zudem sei erwähnt, dass die Veröffentlichung von wissenschaftlichen Arbeiten auf der Grundlage der sprachsystematisch-orientierten und situationskontextbezogenen Theorie des Registers nicht endet, obwohl seit der Beendigung der ersten Phase des ESP schon ein beträchtlicher Zeitraum vergangen ist. Diese Gegebenheit zeigt Gültigkeit der dargestellten Theorien im gegenwärtigen Berufs- und Fachsprachenunterricht. Wie man im nachfolgenden Abschnitt sehen können wird, haben uns einige der angesprochenen Arbeiten als methodologische Grundlage zur Forschung und zur didaktischen Aufbereitung der im Rahmen dieses Beitrags behandelten Ausgangstexte gedient.

Der Theorie des Registers liegen verschiedene strukturalistische Textanalysearten zur Untersuchung und Systematisierung der lexikalisch-syntaktischen Strukturen, des Gebrauchs und des pragmatischen Kontextes einer sprachlichen Varietät zugrunde, wie $z$. B. die in dieser Arbeit dargestellten Analysen.

Im Rahmen dieses Beitrags wird die Theorie des Registers von zwei Perspektiven aus implementiert: einer linguistikbezogenen Perspektive und einer kontextuellen Perspektive. Die erste Studienperspektive legt ihren Akzent auf das sprachstrukturelle Niveau. Nach der herrschenden Auffassung hat diese die Identifizierung der häufigsten lexiko-syntaktischen Prozesse in einer Fachsprache zum Ziel. Ihre Anwendung wird durch zwei verschiedene Textanalysen in die Praxis umgesetzt: eine lexikalische Analyse und eine syntaktische Analyse.

Nach dem Sprachforscher Alcaraz Varó (2000: 198) beschäftigt sich jene lexikalische Analyse mit der semantischen (Fachwortschatz, halbfachspezifischer Wortschatz und allgemeiner Wortschatz) und der morphologischen Lexikologie (Zusammensetzung, Ableitung, Ausdruckskürzung, Kontamination, Konversion Lehnwörter, etc.).

Die erwähnte syntaktische Analyse lässt sich hauptsächlich mittels folgender Parameter durchführen: Tempus, Personalform, Genus Verbi (Aktiv/Passiv), Modus (Indikativ/Konjunktiv/ Imperativ), Art des Satzbaus und Art der Satzglieder (Dudley-Evans/ St. John, 1998). 
Jene kontextuelle Analyseperspektive richtet das Augenmerk auf die kontextuelle Niveaustufe, in der Register zustande kommen. Bibers (1994) Untersuchungsverfahrensmodell bildet den Ausgangspunkt dieser Analyseperspektive. Dieser Sprachforscher schlägt einen Analyserahmen zur Identifizierung folgender sozialer, pragmatischer, funktioneller und situationsbedingter Registerfaktoren vor:

- Pragmatische Eigenschaften der Kommunikationsteilnehmer: A) Absender: Individuell/kollektiv institutionell oder betrieblich. B) Adressat (en): 1) der selbe Absender /andere Adressaten; 2) Individuell/ kollektiv

- Beziehungen zwischen dem Absender und dem Rezipienten: A) Position, Status und Autorität des Absenders und des Rezipienten in den sozialen Beziehungen: Beziehungen zwischen Unbekannten, Beziehungen zwischen Bekannten, Beziehungen zwischen Erwachsenen und Kindern, Beziehungen beruflicher Art, Rangbeziehungen, Beziehungen zwischen Familienmitglieder, etc. B) Interaktionsgrad: hoch/moderat/nichtig.

C) Persönlichkeitsbezogene Beziehungen: Respekt, Furcht, Verwandtschaft, Feindschaft, Freundschaft, etc.

- Kommunikative, räumliche und chronologische Merkmale des Sprachverwendungskontextes: A) Merkmale des kommunikativen Raumes: 1) privat /öffentlich; 2) Domäne: Geschäfts- und Berufszweig, Bildungsbereich, Regierungsund gesetzlicher Bereich, häusliche und persönlichkeitsbezogene Sphäre, Religion, Kunst und Unterhaltung, andere. B) Art des von den Kommunikationsteilnehmern geteilten Raumes: geteilt/ nicht geteilt (die Kommunikationsteilnehmer haben keinen physischen Kontakt, wie etwa bei der Kommunikation per Telefon oder via Internet). C) Merkmale der Kommunikationszeit: direkt/indirekt.

- Produktionsmerkmale: A) Kommunikationsmodus: schriftlich oder gesprochen; Zeichensprache oder Mischung aus verschiedenen Datenübertragungsmodi bzw. Kommunikationsarten. B) Permanenzgrad: aufgespeichert/ 
transitorisch. C) Ubertragungsmittel: 1) Falls gespeichert: Papier, elektronische Datei, andere Speichermedien; 2) Falls transitorisch: von Angesicht zu Angesicht, Telefon, Rundfunk, Fernsehen, andere transitorische Ubertragungsmittel; 3) Publiziert oder unveröffentlicht. D) In eine umfassendere Texteinheit eingebettet: ja/nein.

- Zweck und Absichtlichkeit: A) Überzeugung oder Verkauf: hoch / moderat / niedrig/ gleich null. B) Informationsvermittlung: hoch / moderat / niedrig/ gleich null. C) Unterhaltung / Belehrung: hoch / moderat / niedrig/ gleich null. D) Ausdruck persönlicher Gefühle: hoch / moderat / niedrig/ nichtig. E) Verbesserung der interpersönlichen Beziehungen: hoch / moderat / niedrig/ nichtig. F) Äußerung von Haltungen: hoch / moderat / niedrig/ nichtig. G) Ausdruck von Meinungen: hoch / moderat / niedrig/ nichtig. H) Äußerung anderer Absichten: hoch / moderat / niedrig/ nichtig.

- Topic: A) Fachlich oder allgemein. B) Fachlicher Grad von: hoch/moderat/niedrig/ nichtig. C) Gegenstandsbereich: Finanz, Wissenschaften, Religion, Politik, Sport, Rechtswesen, Menschen, alltägliche Aktivitäten, etc.

- Natur der beschriebenen oder erzählten Ereignissen/Gegebenheiten: real oder irreal, nachgestellt, spekulativ, Mischung aus mehreren Beschreibungsarten.

\section{SPRACHLERNSTRATEGIEN}

Unter Sprachlernstrategien versteht man die Methoden, die der Sprachlernende zum Zweck eines besseren Erwerbs der Fremdsprache verwendet. Diese bestehen in der Anwendung von Taktiken für den Erwerb von Sprachstrukturen und Wortschatz sowie der dabei verwendeten Vorgehensweise.

Strategien zum Erwerb von Fremdsprachen haben eine lange Tradition in der Geschichte der Menschheit und es liegen heutzutage zahlreiche Klassifizierungen davon vor. Rebecca Oxfords (1990) Kategorisierung ist nach der vorherrschenden Auffassung eine der 
wichtigsten, unter anderem da sie als Basis für weitere Kategorisierungen diente. Darin werden neben den Lernstrategien zur direkten Verarbeitung der Sprache (Direkte Lernstrategien) jene Lernstrategien ausführlich betrachtet, welche die Planung des Lernprozesses der Fremdsprache beinflussenden reflexiven, affektiven und sozialen Faktoren umfassen (Indirekte Lernstrategien) (17).

$\mathrm{Zu}$ der Gruppe der direkten Lernstrategien gehören die Memorierungsstrategien (z. B. Retention von Grammatikregeln oder von Vokabular, Kreation mentaler Referenzobjekte, Einsatz von Bildern und Geräuschen), die kognitiven Lernstrategien (z. B. Folgerungen aus den sprachlichen Phänomenen, Erschließung von unbekannten Wörtern durch den Sprachzusammenhang, wichtige Informationen herausnehmen) und die Kompensationsstrategien ( $z$. B. Umschreibung von nicht verfügbaren sprachlichen Elementen in der Fremdsprache) (Oxford, 1990: 17).

Die indirekten Lernstrategien lassen sich in folgende Strategien gruppieren: die metakognitiven Strategien (z. B. Nachdenken über den Fremdsprachenlernprozess, Führung eines Sprachlerntagebuchs, Bewertung des in einem bestimmten Zeitabschnitt neu erworbenen Wortschatzes), die affektiven Strategien (z. B. Beseitigung der Sprechangst und Förderung des Selbstvertrauens beim Sprechen) und die sozialen Strategien (z. B. kompetente Gesprächspartner der Zielsprache nach den unbekannten Sprachelementen fragen, Assimilation der interkulturellen Aspekte der zu erlernenden Sprache und gegenseitiger Austausch von Sprachlernerfahrungen mit anderen Lernenden) (Oxford, 1990: 17).

Ebenso ist hierbei die Kategorisierung von Glaboniat et al. (2002) zu nennen. Neben den gerade erwähnten Lernstrategien umfasst diese Kategorisierung eine Gruppe von indirekten Lernstrategien zur Überwindung von fremdsprachigen Situationen: die Kommunikationsstrategien. Bei diesen Strategien geht es um die Ausarbeitung von kommunikationsorientierten Plänen zur Produktion von in bevorstehenden fremdsprachigen Kommunikationssituationen angeforderten Sprachelementen und zur Überwindung der dabei auftretenden Hindernisse. Diese speziellen 
Strategien lassen sich in der Regel nach dem folgenden Handlungsschema einsetzen:

\begin{tabular}{|l|l|}
\hline Planung & \multicolumn{3}{|c|}{ Sich vorbereiten auf die kommunikative Aufgabe } \\
\hline $\begin{array}{l}\text { Durchführung } \\
\text { Produktion }\end{array}$ & $\begin{array}{l}\text { Durch den Einsatz von Techniken die } \\
\text { kommunikative Aufgabe effizient und erfolgreich } \\
\text { meistern }\end{array}$ \\
\hline Kontrolle & $\begin{array}{l}\text { Vorannahmen und Wirkung der eingesetzten } \\
\text { kommunikativen Mittel und Techniken überprüfen }\end{array}$ \\
\hline Reparatur & $\begin{array}{l}\text { Falls nötig: Vorannahmen revidieren, } \\
\text { Missverständnisse klären (51) }\end{array}$ \\
\hline
\end{tabular}

Bei den in dieser Arbeit auf der Theorie des Registers zurückgehenden Sprachaktivitäten sollen die Lernenden Lernstrategien aus den gerade erwähnten Kategorisierungen von Oxford und Glaboniat et alli anwenden. Diese Sprachaktivitäten werden in der Unterrichtsphase präsentiert.

\section{UNTERSUCHUNGSPHASE}

Die hier dargelegte Theorie des Registers ist auf einen Textkorpus aus zwölf Dienstleistungsbetriebswebseiten der Wellness- und Gesundheitstourismusbranche angewandt worden ${ }^{3}$. Die Auswahl dieser authentischen Textmaterialien erfolgte unter Beachtung der Sprachbedürfnisse der Lernenden. Diese Kursmaterialien enthalten zahlreiche Sprachelemente von großem Nutzen für ihre gegenwärtigen oder zukünftigen Berufstätigkeiten im gerade erwähnten Tourismussektor.

Solche Ausgangstexte werden im Sprachfach Aufbaukurs Modernes Deutsch in der Tourismusbranche verwendet. Dabei handelt es sich konkret um ein Sprachpraxisfach im sechsten Semester des

${ }^{3}$ Siehe Bibliographie. 
Tourismusstudiums an der Universität Almería, an dem Studenten der Studiengänge Tourismus, Gesundheits- und Krankenpflege und Physiotherapie teilnehmen. Diese Sprachlernenden besitzen ausreichende Sprachkenntnisse (Sprachniveau A2 des Europäischen Referenzrahmens der Sprachen) zur erfolgreichen Bewältigung der

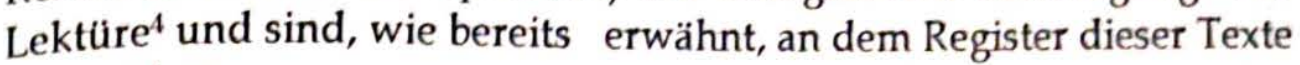
interessiert.

Im Folgenden werden die Forschungsergebnisse der Theorie des Registers auf den ausgesuchten Web-Sites vorgestellt.

A) Lexikalisch-semantische Wortschatzanalyse:

Im analysierten Textkorpus lässt sich eine große Präsenz des allgemeinen Wortschatzes (gelingen, super, lecker, traumhaft, Landschaft, usw.) und des semitechnischen Wortschatzes erkennen. Innerhalb der zweiten Hauptkategorie überwiegen die Lexikbestände aus der Tourismus- (Kurzurlaub, Hotelaufenthalt, Vollpension, Freiraum, Dinner, usw.) und Wellnesssprache (Bio-Sauna, Lounge-Ecke, Teebar, Wellnesshotel, Ganzkörpermassage, Fußreflexzonen-Massage, usw.).

B) Lexikalisch-morphologische Wortschatzanalyse:

Die häufigsten Wortbildungsressourcen in der Gesamtheit der Ausgangstexte sind die Nominalkomposition ${ }^{5}$ (Gesundheitserlebnis, Wellnesshotel, Behandlungsschwerpunkt, Teebar, Erlebnispool, usw.), Komposition aus Substantiv und Adjektiv bzw. Partizip (Kurzurlaub, sonnenverwöhnt, naturbelassen, Vollpension, Freiraum, usw.), Komposition mit Bindestrich (Salz-Ruhebecken, Bio-Solarium, Gesundheits-Programm, Ganzkörper-Kräuterpeeling, KräuterCremepackung, usw.), Komposition aus deutschem und Fremdwort (Sole-Trinkkur, Spa-Erlebnis, Gesundheitsprophylaxe, Strandfeeling, Silence-Aromamassage, usw.), Ableitung mit Suffixen (Wirkung,

${ }^{4}$ Deren Lektüre kann sogar durch Lernende auf einem niedrigen Deutschniveau (Anfängerniveau A 1.2 des Europäischen Referenzrahmens der Sprachen) vorgenommen werden.

${ }^{5}$ Dieses morphologische Wortbildungsmittel ist sehr üblich in Werbetexten wie den hier vorherstellten Ausgangstexten. Durch die langen zusammengesetzten Sprachelemente versucht der Unternehmer den größtmöglichen Informationsgehalt im kleinsten Raum und auf die schnellstmögliche Weise darzustellen. 
whtersdiedlich, kostenlos, Mitgliedschaft, Behaglichkeit, usw.), Ableitung durch Halbpräfixe (erwärmen, verdampfen, erleben, erdenklich, Einnchtung, usw.) und Übernahme von Fremdwörtern (Tepidarium, Spa, (heck, Revital, Shape, usw.).

C) Syntaxanalyse ${ }^{6}$ :

Tempus: Das Indikativ Präsens ist die dominierende Zeitform im Text: Die Wellness-Oase im Strandhotel Georgshöhe bietet... (<http://www.georgshoehe.de>); Durch die starke Meeresbrandung erhält... (<http://www.oceano.de〉); Dabei verbindet er... (<http://www.wartherhof.at>); Beide führen... (<http://www.westingrandmunich.com>); Qi Gong besteht aus... (<http://www.schwarz.at>).

Personalformen: Die konjugierten Verbformen in der dritten Person Singular und in der Höflichkeitsform dominieren in den Texten.

Personalformen in der dritten Person Singular: Die moderne Technik in $\begin{array}{llr}\text { Ihrem Tagungshotel München } & & \text { macht... } \\ \text { (<http://www.westingrandmunich.com>); } & \text { So } & \text { ist... }\end{array}$ (<http:// www.strandhotel-kurhaus-juist.com>); Thermen Bussloo ist... (<http://www.thermenbussloo.nl>); Hier trifft... (<http://www.oceano.de/hotel.html>); Wer in Österreich nach Wellness sucht... (<http://www.schwarz.at>).

Personalformen in der Höflichkeitsform: Durch beste Verkehrsanbindungen sind Sie... (<http:// www.westingrandmunich.com>); Sie reservieren... (<http://www.thermenbussloo.nl>); Als Gast des OCÉANO Vitality Hotels wohnen Sie... (<http://oceano.de〉); Denn im SunWelly Spa finden Sie... (<http://www.schwarz.at>); Genießen Sie... (<http://www.schoenruh-seefeld.com>).

Die erste Person Plural ist auch nicht zu übersehen. Diese Verbform befindet sich in fast allen analysierten Websites: Nehmen wir

\footnotetext{
6 In diesem Untertextabschnitt halten wir es für angebracht, die Quellen der angesprochenen Zitatbeispiele hinzuzufügen (außer den Beispielzitaten, die nur aus einem Wort bestehen), da diese größtenteils aus Sätzen oder Satzgliedern aus mehr als zwei Sprachelementen bestehen und daher Urheberrechten unterstehen.
} 
an... (<http://strandhotel-kurhaus-juist.com>); Unter dem Link "Häufig gestellte Fragen" haben wir... "(<http://www.thermenbussloo.nl>); Als Familienbetrieb bauen wir... (<http://www.wartherhof.at $>$ ); Hier verwöhnen wir... (<http://www.schwarz.at>); Deshalb sind wir... (<http://schoenruhseefeld.com $>$ ).

Modus (Indikativ/Konjunktiv/Imperativ): Im Korpus überwiegt der Indikativ: In intimer Atmosphäre führen wir... (<http://www.georgshoehe.de>); Den intensiven therapeutischen Effekt des warmen Meerwassers wenden wir... (<http://oceano.de $>)$; In einem der SAMINA Schlafsysteme stecken... (<http://www.wartherhof.at>); Es stehen... (<http://www.westingrandmunich.com>); Im Rahmen der SCHWARZ-Verwöhnpension (<http://www.schwarz.at>).

genießen

Sie...

Die Imperativform fällt ebenso auf. Diese Modusform tritt in den meisten analysierten Ausgangstexten auf: Verbinden Sie... (<http://www.georgshoehe.de>); Dann rufen Sie... (<http://strandhotel-kurhaus-juist.com>); Tauchen Sie ein... (<http://www.georgshoehe.de>); Wählen Sie... (<http://www.thermenbussloo.nl>); Fühlen Sie sich... (<http://www.oceano.de>).

Genus Verbi (Aktiv/Passiv): Der Großteil der Korpustexte steht im Genus Aktiv: Die vom Hotel aus frei zugängliche Meeres-Piscina ermöglicht... (<http://www.oceano.de>); Ein gesunder Schlaf ist... (<http://www.wartherhof.at>); Saunadörfle bietet... (Ibid.); Menschen mit regelmäßigem Qi Gong Training entwickeln... (<http://www.schwarz.at/de>).

Genauso finden sich Passivgefüge im Großteil aller Webseiten: Alle Anwendungen werden von ausgebildeten, fachkundigen Therapeuten durchgeführt (<http://www.westingrandmunich. com>); Eine "Offene Badekur" wird von vielen Krankenkassen unterstützt (<http://www.georgshoehe.de>); Reservierungen werden unter Tel. +31 $\begin{array}{lllll}(0) 55 & 368 & 26 & 40 & \text { entgegengenommen }\end{array}$ (<http://www.thermenbussloo.nl>); So werden Ihre natürlichen Selbstheilungskräfte aktiviert (<http://www.oceano.de>); Das Alpenresort Schwarz wurde [...] gekürt (<http://www.schwarz.at〉). 
Art des Sitzbaus. Da es sich hierbei um Werbetexte handelt, ist die Vielzahl einfacher Gefügen höher als die der komplexen. Jedoch enthalten einige Webseiten eine nennenswerte Menge von zusammengesetzten Sätzen.

Einfache Gefüge: Kokosnuss-Öl pflegt die Haut und verwöhnt sie mit wertoollen Nährstoffen (<http://www.georgshoehe.de>); Direkt aus dem Meer wird frisches Meerwasser in unser Medical Spa gepumpt (<http://www.oceano.de>); Ein Traum wird wahr! (<http://www.wartherhof.at>); Lemen Sie die grünen Seiten Münchens aus einem neuen Blickwinkel kennen (<http://www.westingrandmunich.com>); Mitten in unserem Wellness-Hotel in Miening bietet Ihnen die Privatklinik am Sonnenplateau Behandlungen aus (<http://www.schwarz.at>).

Alle analysierten Websites enthalten komplexe sprachliche Gebilde oder Hypotaxen. Der Großteil davon entfällt auf die Relativsätze. Diese Satzart ist in fast allen diesen Texten vorhanden: Verwöhnen Sie Ihre Seele mit dem Besten, was Wellness zu bieten hat (<http://www.westingrandmunich.com>); Gerne beraten wir Sie in entspannter Atmosphäre, welcher dieser (individuellen) Wege besonders geeignet ist (<http://www.oceano.de>); Deshalb möchten wir an dieser Stelle nicht zu viel verraten - sondern Ihnen nur einen kleinen Eindruck geben, was Sie in unserem Wellnesshotel und Resort in Tirol alles enwartet (<http://www.schwarz.at>); Freuen Sie sich auf die Annehmlichkeiten, die Innen nur ein 4 Sterme Wellnesshotel bieten kann (<http://www.schoenruh-seefeld.com〉); Die Wellness und Sport Angebote im Wellnesshotel ZUGBRÜCKE sind sehr vielfältig und reichen von klassischen oder trendigen Behandlungen für Gesicht und Körper über Thalasso und Ayurveda bis hin zu Shiatsu und vielfältigen Mental-sowie Aktivprogrammen, die stets von bestens ausgebildetem und erfahrenem Personal durchgeführt werden (<http:// www.zugbruecke.de>).

Auf die gleiche Weise, obwohl in geringerem Maß, enthalten die meisten Websites des analysierten Textkorpus zumindest einen oder zwei Infinitivsätze: Wir behalten uns vor, während aller Feiertage eine Mindestbelegdauer festzulegen (<http://www.jammertal.de>); Es ist auch möglich eine Behandlung in der Private Wellness zu erhalten (<http://www.thermenbussloo.nl>); Ganz gleich, was Sie in die 
bayerische Landeshauptstadt zieht, alle 627 Zimmer und Suiten empfangen Sie im Westin Stil und laden sofort dazu ein, den Alltag zu vergessen (<http://www.westingrandmunich.com>); Unsere erfahrenen Ärzte und Therapeuten unterstützen Sie gern dabei, eine auf Ihre körperlichen und seelischen Bedürfnisse maßgeschneiderte Therapie zu wählen (<http://www.oceano.de >); Damit bieten wir Ihnen mehrere Möglichkeiten sich ein möglichst objektives Bild unseres Wellness Hotels in Vorarlberg zu machen und mit anderen Gästen zu kommunizieren (<http://www.wartherhof.at>).

Art der Satzglieder: Im Ausgangstextkorpus treffen wir auf Satzglieder verschiedener Komplexität. Diese variieren zwischen Satzgliedern aus einem Wort bis hin zu jenen aus mehr als drei Sprachelementen?.

Einwortsatzglieder: Thalasso, Raum, Ihnen, Sie, einfach.

Zweiwortsatzglieder: der Stoffwechsel (<http://www.oceano.de>), kulinarische Höhenflüge (<http://www.wartherhof.at $>)$, im Pool (< http://www.westingrandmunich.com $>), \quad$ im Sommer (<http://www.schwarz.at>), Die Kuppel (<http://wrwo.strandhotelkurhaus-juist.com>).

Dreiwortsatzglieder: in freier Natur (<http://www.schwarz.at $>$ ), für die Anwendungen (<http://www.oceano.de>), In einzigartiger Lage (<http://www.strandhotel-kurhaus-juist.com>), ein Paar Laufschuhe (<http://www.westingrandmunich.com>), Panoramaschwimmbecken mit Gegenstromanlage (<http://www.wartherhof.at>).

Satzglieder mit mehr als drei Spracheinheiten: ein ganzheitliches Verwöhnprogramm für Körper und Seele (<http://www.georgshoehe.de $>)$, mit seinem enormen Potenzial an Spurenelementen (<http://www.oceano.de $>)$; in unserem 4 Sterne Hotel in Vorarlberg (<http://www.wartherhof.at>); direkt vor der Tür (<http://www.schwarz.at>), jeden nur erdenklichen Komfort (<http://www.westingrandmunich.com>).

\footnotetext{
${ }^{7}$ Wie bei den zusammengesetzten Wörtern werden diese komplexen Satzglieder benutzt, um zahlreiche Informationen auf eine schnellere und wirksamere Weise auszudrücken.
} 
D) Pragmatische Eigenschaften der Kommunikationsteilnehmer:

Absender: Der Absender der „Website des gesundheitstouristischen Betriebs" ist in der Regel von pluralischer und betrieblicher Natur. Dieser setzt sich aus einigen der folgenden Akteure zusammen, die in dem jeweiligen Dienstleistungsbetrieb arbeiten oder in ihn investieren: das Führungsteam, die Betriebsmitglieder, die Aktionäre, die Eigentümer, die Arbeiter, etc. Wie hinlänglich bekannt, sind diese an der erfolgreichen Werbung für ihr Geschäft interessiert, weshalb sie direkt oder indirekt die Verantwortlichen für die Produktion dieser Spracheinheit sind.

Rezipient(en): 1) Einer selbst/andere: Wie bei jedem Werbetext, ist die "Website des gesundheitstouristischen Betriebs" nicht an "einen selbst" adressiert, sondern an andere Rezipienten. 2) Individuell/pluralisch: Der Rezipient ist pluralischer Natur, da diese Spracheinheit beabsichtigt, beim größtmöglichen Publikum anzukommen.

E) Beziehungen zwischen dem Absender und dem Rezipienten:

Soziale Beziehungen: Beide Akteure im Kommunikationsvorgang sind einander unbekannt. Zudem verfügt der Absender über weniger Macht. Aus diesem Grund entsteht eine hierarchische Beziehung zwischen "Vorgesetzter" und "Untergebenem". Der erste Kommunikationsakteur ist der "Verkäufer" von den in der Website des gesundheitstouristischen Betriebs angebotenen Produkten und handelt daher als ein "Untergebener" seines „Vorgesetzten", des Rezipienten, des potenziellen Kunden und Verbrauchers solcher Produkte.

Interaktionsgrad: In der Kommunikation über die Website des gesundheitstouristischen Betriebs kann nur eine moderate Interaktion zwischen dem Absender und dem Rezipienten entstehen, da die in ihr behandelten Themen grundsätzlich von Informationen, Verkäufen oder Reservierungen der angebotenen Produkte handeln.

Persönliche Beziehungen: Die Beziehungen von gegenseitigem Respekt zwischen beiden Kommunikationspartnern dominieren in dem Kommunikationsprozess über die angesprochene Spracheinheit. 
Wie man bei der Gesamtheit der analysierten Ausgangstexte feststellen kann, werden solche Beziehungen durch soziopragmatische Konventionen in die Tat umgesetzt (und zwar durch den Einsatz der Höflichkeitspronomen).

F) Kommunikative, räumliche und chronologische Merkmale des Sprachverwendungskontextes:

Merkmale des kommunikativen Raumes: 1) privat/öffentlich: Der Gegenstand dieser Spracheinheit ist im öffentlichen Kommunikationsraum eingerahmt. Diese Tatsache ergibt sich aus ihrem reinen Werbungscharakter. 2) Domäne: Die Geschäfte bilden ihre Hauptdomäne.

Art des von den Kommunikationsteilnehmern geteilten Raumes: Da es sich hierbei um einen virtuellen Text handelt, bildet die Website des gesundheitstouristischen Betriebs einen nicht geteilten Raum.

Merkmale der Kommunikationszeit: Die Kommunikation über die Website des gesundheitstouristischen Betriebs ist zeitversetzt. Die Rezipienten können sich lediglich schriftlich mit dem Kommunikator verständigen.

G) Produktionsmerkmale:

Kommunikationsmodus: Diese Spracheinheit des Untersuchungsgegenstandes kombiniert schriftliche Zeichen mit ikonischen Elementen. In der Regel entsprechen die illustrierten Elemente den schriftlichen Beschreibungen und verschaffen einen attraktiven Überblick über die touristische Gesundheitswellnessanlage sowie deren angebotenen Dienstleistungen.Permanenz: Die analysierte Spracheinheit ist ein permanenter Text.

Übertragungsmittel: 1) Falls aufgespeichert: A) Permanent oder transitorisch: Dieses Genre befindet sich auf einem permanenten elektronischen Format. 2) Publiziert/unveröffentlicht: Der besagte Ausgangstext ist publiziert.

In eine umfassendere Texteinheit eingebettet: Die Website des gesundheitstouristischen Betriebs kann einen unabhängigen Text bilden 
oder Bestandteil anderer Spracheinheiten sein wie, unter anderem, der Tourismus-Website.

H) Ziele und Absichten:

Uberzeugung oder Verkauf: Wie bei jedem Geschäftstext bestehen die Hauptziele der Website des gesundheitstouristischen Betriebs in der Üherzeugung und in dem Verkauf.

Informationsvermittlung: Der besagte Ausgangstext versucht, zahlreiche Auskünfte über die Dienstleistungen des werbenden Dienstleistungsbetriebs zu vermitteln.

Unterhaltung: Dieser Werbetext versucht, den Leser $\mathrm{zu}$ unterhalten, damit dieser sich für die darin angebotenen Produkte und Leistungen interessiert.

Belehrung: Nichtig.

Ausdruck persönlicher Gefühle: Nichtig.

Verbesserung der interpersönlichen Beziehungen: Nichtig.

Äußerung von Haltungen: Nichtig.

Ausdruck von Meinungen: $\mathrm{Da}$ es sich hierbei um einen Geschäftstext handelt, werden in der besagten Website positive Meinungen über die angebotenen Dienstleistungen geäußert.

I) Topic:

Fachlich (specialized) oder allgemein (general): Dieses Niveau ist allgemeinsprachlich und teilweise spezialisiert, denn obwohl dieser Text von einem Dienstleistungsbetrieb aus der WellnessTourismusbranche hervorgebracht worden ist, wendet er sich an das allgemeine Publikum.

Fachlicher Grad: Der Fachlichkeitsgrad der analysierten Gattung ist moderat, denn deren Dienstleistungen werden einem Publikum ohne Fachkenntnisse über den Gesundheitsbereich angeboten.

Thematisches Feld: Diese Textsorte gliedert sich im touristischen Gesundheits-Wellnessdienstleistungsfeld ein. 
J) Natur der beschriebenen oder erzählten Ereignisse/Gegebenheiten:

Die Website des gesundheitstouristischen Betriebs geht in der Regel auf wirkliche Gegebenheiten zurück. Allerdings kann diese Spracheinheit einige fiktive Fragmente enthalten, um ihre Informationen attraktiver zu gestalten.

\section{UNTERRICHTSPHASE}

In dieser Phase stellen wir eine Serie von pragmatikbezogenen Aktivitäten zur Didaktik der grundsätzlichen sprachlichen und soziokontextuellen Merkmale der Website des gesundheitstouristischen Betriebs vor. Diese Aktivitäten gehen von folgenden Faktoren aus: den erwähnten Variablen der Registeranalyse und Lernstrategien sowie den in den vorangegangenen Textabschnitten dargestellten Forschungsergebnissen ihrer Applikation.

Aufgaben zum Erwerb von Sprachelementen und zur Einsetzung von Memorierungsstrategien:

1. Bilden Sie aus den folgenden Verben Substantive ${ }^{8}$ : informieren, leiden, einnehmen, begleiten, entwickeln, verletzen, absagen, stornieren, berechnen, auswählen, einfallen, schöpfen, inspirieren, genießen, arbeiten, beraten.

2. Bilden Sie aus den folgenden Substantiven Verben': die Entspannung, die Ausgeglichenheit, die Gestaltung, die Behandlung, die Anwendung, das Angebot, die Beschwerde, die Behinderung, die Erholung, das Schwimmbad, die Vergabe, die Bekleidung, die Haftung, die Reservierung, die Anwendung, die Rasur.

${ }^{8}$ Diese Aufgabe dient zur Praxis folgender Memorierungsstrategie. Wenn man ein Verb lernt, kann man diese Lerngelegenheit dazu nutzen, um das Substantiv mit dem gleichen Grundmorphen zu lernen.

9 Bei dieser Aufgabe handelt es sich um folgende Memorierungsstrategie: Das Lernen eines Substantivs zusammen mit dem Verb mit dem gleichen Grundmorphen. 
3. Bilden Sie zusammengesetzte Wörter ${ }^{10}$ :

\begin{tabular}{|l|l|}
\hline die Gesundheit, das Konzept: & die Natur, die Kraft: \\
das Meer, das Rauschen: & immun, das Organ: \\
der Fischer, das Dorf: & immun, Abwehr: \\
die Küste, die Formen: & der Körper, die Zelle: \\
die Freizeit, die Möglichkeit: & der Nerv, die Zelle: \\
die Behandlung, das Ziel: & der Bauch, die Behandlung: \\
die Behandlung, das Programm: & die Therapie, der Verlauf: \\
der Genuss, der Faktor: & der Schmerz, die Linderung: \\
\hline
\end{tabular}

4. Markieren Sie 10 wellnessbezogene Wörter auf der folgenden Website: www zentral.it ${ }^{11}$

5. Markieren Sie 10 tourismusbezogene Wörter auf der folgenden Website: www.zugbruecke.de

6. Schreiben Sie aus den folgenden Websites je 10 Wörter aus der Wellnesssprache heraus: www.wartherhof.de; www.georgshoehe.de; www.wellnesshotels-deutschland.de/jammertal.

7. Ordnen Sie die nachfolgenden Wörter der Tourismussprache oder der allgemeinen Sprache zu:

\begin{tabular}{|l|l|l|l|}
\hline Behandlungssuite & Kissenbar & Stromkreis & Schlaf \\
Doppelzimmer & Übernachtung & Einzelzimmer- & Biergartenpass \\
Programmzeit & Doppelzimmer & Zuschlag & Mindestbelegdauer \\
Junge & Kulinarisch & Doppelzimmer- & Schlemmerbüffet \\
Speisekarte & Dessert- & Belegung & Verkehrsanbindung \\
Computer & Klassikern & Wagen & Allergikerkost \\
\hline
\end{tabular}

\footnotetext{
${ }^{10}$ Diese Aufgabe bedarf einer näheren Erklärung. Wenn die Studierenden lernen, zusammengesetzte Wörter zu lesen, werden sie deren Bedeutung leichter schlussfolgern und sie sich besser merken.

1 Die Aufgaben 4 bis 8 dienen zur Memorierung von tourismus- und wellnessbezogenem Wortschatz.
} 


\begin{tabular}{|c|c|c|c|}
\hline $\begin{array}{l}\text { Service } \\
\text { Straßenbahn } \\
\text { Lebensmittel } \\
\text { Halbpension }\end{array}$ & $\begin{array}{l}\text { Vorspeise } \\
\text { Schule } \\
\text { Feiertag } \\
\text { Spargelsalat } \\
\text { Apfel }\end{array}$ & $\begin{array}{l}\text { Ausflugs-Tipp } \\
\text { Forellenkaviar } \\
\text { Dessertbüffet } \\
\text { Tür }\end{array}$ & $\begin{array}{l}\text { Meeresfrüchtesalat } \\
\text { Tisch } \\
\text { Geflügelspezialität } \\
\text { Reservierung }\end{array}$ \\
\hline
\end{tabular}

8. Welche der nachfolgenden Wörter gehören Threr Meinung nach zum Wellnesswortschatz?:

\begin{tabular}{|l|l|l|l|}
\hline Gesundheitserlebnis & Ruhe & Obst & Landeshauptstadt \\
Wellness-Oase & Dampfnebelgrotte & Elektro- & Beauty-Bad \\
Bademantel & verwöhnen & Lypolyse & Alltag \\
Badebistro & Aroma-Massage & Stromkreis & Bio-Sauna \\
Wellness & Familie & Balneologie & Dampfnebelgrotte \\
Entspannungstag & Body-Scan & Schlaf & Raum \\
Spielzeug & Aufenthalt & Caldarium & Elektro-Lypolyse \\
Wasserbettmassage & Day Spa & Freude & Kreativität \\
Solarium & Entschlackungskur & Dermabrasion & Erdwallsauna \\
Cleopatrabad & Detail & Uhrzeit & Frühstück \\
& & Leidenschaft & \\
\hline
\end{tabular}

Aufgaben zum Erwerb von Sprachelementen und zur Einsetzung von kognitiven Lernstrategien zur Schlussfolgerung von Grammatikphänomenen:

1. Suchen Sie in den von Ihnen ausgewählten Texten zehn Wörter, die durch Ableitung durch Präfixe gebildet sind und identifizieren Sie das Präfix und den Wortstamm. Existiert diese Wortbildungsressource in Threr Sprache? ${ }^{12}$

2. Suchen Sie in den von Ihnen ausgewählten Texten zehn Wörter, die durch Ableitung durch Suffixe gebildet sind und identifizieren Sie das Suffix und den Wortstamm. Existiert dieses Wortbildungsphänomen in Ihrer Sprache?

12 Bei den Aufgaben eins bis sechzehn sollen konkret kognitive Lernstrategien zur Verarbeitung der wichtigsten Wortbildungsverfahren im Deutschen angewandt werden. 
3. Finden Sie die Wortbildungsressourcen in den nachfolgenden wistern heraus: befeuchten, enthalten, geräumig, unvergleichlich, kostenlos, abwechslungsreich, Erwartung, entspannend, ehrlich, hochwertig, heimelig, köstlich, Leckerei, ausgleichend, beruhigend, erfrischend, reichhaltig, abreiben, durchblutet, göttlich, versorgen, unverwechselbar, unkompliziert, Gastlichkeit, unvergesslich.

4. Suchen Sie in den von Thnen ausgewählten Texten zehn Wörter, die durch Komposition gebildet sind und zerlegen sie in ihre Bestandteile. Wird dieses Wortbildungsverfahren in Ihrer Muttersprache so oft eingesetzt wie in der deutschen Sprache?

5. Zergliedern Sie folgende Komposita in ihre Bestandteile: Lichtdurchflutet, Ernăhrungsgewohnheit, Frühdiagnostik, Erlebnisduschen, Körperbehandlung, Milchölbad, Kräutersauna, Krankengymnastik, Reflexzonenmassage, Kaffeerösterei, Kosmetikanwendung, Maltherapie, Nahrungsergänzung, Naturkosmetik, Gesundheitswissen, Körperpeeling, Reduktionsdiät, Rückenschule, Muskelentspannung.

6. Finden Sie alle möglichen Wortbildungsphänomene in den nachfolgenden Wörtern heraus: Hausgemacht, Ernährungsberatung, Meditations-Therapie, Verdauungsapparat, Wundheilungsfördernd, Mineralsalzbehandlung, Regeneration, Reflexzonenmassage, Liegebike, ganzheitlich, Schönheitskonzept, Traumhaft, Nahrungsmittelunverträglichkeit, Fremdenverkehrstreiben, Stellplatz, Tiefgarage, Radfahren, Mountainbiken, Sonnenverwöhnt, wohlsortiert, wunderschön, handwerklich.

7. Suchen Sie 4 komplexe Satzkonstruktionen aus den folgenden Websites heraus: www.schoenruh-seefeld.com; www.panoramahotel.de.

8. Schreiben Sie fünf Relativsätze aus den folgenden Websites heraus und formulieren Sie eine Regel zu diesem Grammatikphänomen: www.zugbruecke.de, www.schoenruhseefeld.com, www.georgshoehe.de.

9. In welcher Person und welchem Numerus steht der Großteil der konjugierten Verbformen in den von Ihnen analysierten Texten? 
10. Was ist die dominierende Zeitform in den von Thnen analysierten Texten? Suchen Sie Beispiele dafür.

11. Welche Personalformen überwiegen in den von thnen analysierten Texten?

12. Welche Verbmodusformen sind Thnen bei der Lektüre der von Ihnen ausgewählten Texten aufgefallen?

13. Gibt es in den nachfolgenden Websites Passivsätze?: www.wellnesshotels-deutschland.de / georgs-hoehe; westingrandmunich.com.

14. Schreiben Sie vier Passivsätze aus den nachfolgenden Websites heraus und formulieren Sie eine Regel zu diesem Grammatikphänomen: wellnesshotels-deutschland.de / georgshoehe, www.oceano.de.

15. Schreiben Sie 4 Infinitivsätze aus den folgenden Websites heraus und formulieren Sie eine Regel $z u$ diesem Grammatikphänomen: www.zugbruecke.de, www.wellnesshotelsdeutschland.de/jammertal, www.georgshoehe.de.

16. Schreiben Sie 4 Imperativsätze aus den folgenden Websites heraus und formulieren Sie eine Regel zu diesem Grammatikphänomen: www.zugbruecke.de, www.schoenruhseefeld.com/de, www.panorama-hotel.de.

Aufgaben zum Erwerb von Sprachelementen und zur Einsetzung von kognitiven Lernstrategien zur Erleichterung des Lesens in der Fremdsprache:

1. Suchen Sie die Konjunktionen und die Relativpronomen aus den nachfolgenden Textpassagen heraus. Gibt es zusammengesetzte Sätze in den Texten? Wird Ihnen die Lektüre des Textes durch die Ermittlung der Konjunktionen und der Relativpronomen erleichtert? ${ }^{13}$

${ }^{13}$ Bei dieser Aufgabe handelt es sich um die kognitive Strategie zur Identifizierung von Konjunktionen und Relativpronomen. Diese hat zum Ziel, die Lektüre von Texten mit komplexen Sätzen zu vereinfachen. 
Wir wissen, dass es für Sie sehr wichtig ist, Ihr Fitness- und Laufprogramm täglich durchzuführen. Auch wissen wir, dass dies auf Reisen nicht immer ganz einfach ist, nicht zuletzt wegen den strikten Handgepäck-Regeln, die das Mitnehmen von zusätzlichen Schuhen oft verhindern (<http://www.westingrandmunich.com>).

Die Wàrme der Saunakabine führt zu einer angenehmen TiefenEntspannung Wir wissen, dass es für Sie sehr wichtig ist, Ihr Fitnessund Laufprogramm täglich durchzuführen; die folgende Abkühlung löst Nervenreize aus, die eine Erfrischung von Geist und Seele bewirken.

Zudem werden Hormone ausgeschüttet: Schon während des Saunabades setzt eine nachhaltige psychische Erholung ein, die für ein Rundum-Wohlbefinden sorgt (<http://www.georgshoehe.de>).

2. Suchen Sie die komplexen Satzglieder aus den nachfolgenden Websites heraus ${ }^{14}$ :

Die Wellness-Oase im Strandhotel Georgshöhe bietet ein ganzheitliches Verwöhnprogramm für Körper und Seele. In intimer Atmosphäre führen wir Sie schrittweise zurück zu Ihrer ureigenen Balance und völliger Entspannung. Beginnen Sie mit der Suche nach Ausgeglichenheit und schöpfen Sie wieder Kraft, Lebensfreude, Wohlbefinden und Vitalität - auch über Ihren Aufenthalt hinaus (<http:// www.georgshoehe.de>).

Für Weinliebhaber aus aller Welt ist die Moselregion ein wahrer Fund. Denn nur wenige Gegenden produzieren so viele verschiedene Weine, in deren Geschmack sich die Pracht eines ganzen Landstrichs entfaltet. Wir laden Sie herzlich ein, unsere kleine Urlaubswelt kennen zu lernen (<http:// www.panorama-hotel.de>).

3. Gibt es Satzglieder aus mehr als drei Wörtern in den von Ihnen analysierten Texten? Falls ja, schreiben Sie fünf davon heraus.

4. Wozu werden lange Satzglieder in den Texten benutzt? Begründen Sie Ihre Antwort. 14 Die kognitive Strategie zur Interpretation von langen Satzgliedern soll bei den
Aufgaben 2 bis 5 eingeübt werden. 
5. Segmentieren Sie die folgenden Textabschnitte in ihre Satzglieder. Fällt Thnen dann die Lektüre der Texte leichter?

Wir möchten Sie einladen, Ihr ganzheitliches Wohlbefinden im außergewöhnlich exklusiven Ambiente unseres Hauses zu genießen. $O b$ nun der einzigartige Luxus in Meerwasser zu schwimmen, dem Ursprung des Lebens, oder die Seele baumeln lassen in unseren Saunen mit einzigartigem Ausblick auf die bewegte See-Vier-SterneSuperior-Wellness heißt bei uns, wie im siebten Himmel zu sein (<http://www.georgshoehe.de>).

Lassen Sie sich dabei vom Anblick des Atlantiks bezaubern, entspannen Sie sich bei einem Strandspaziergang, in der Blütenpracht unseres Hotelgartens oder beim Genuss mediterraner Köstlichkeiten in unserem Restaurant. Fühlen Sie sich in der kostbaren Zeit Ihres Urlaubs wohl und genießen Sie mit allen Sinnen. Seien Sie unser Gast! (<http://www.oceano.de>)

6. Zergliedern Sie fünf für Sie schwierige lange Komposita in ihre Bestandteile. Verstehen Sie die langen Wörter besser, wenn Sie sie in ihre Bestandteile aufteilen? ${ }^{15}$

Aufgaben zum Erwerb von Sprachelementen und zur Einsetzung von Kompensationsstrategien:

1. Suchen Sie die Ihnen bekannten Fremdwörter (Internationalismen) aus den von Ihnen ausgewählten Texten heraus und beantworten Sie die nachfolgenden Fragen: Welche davon kommen Ihnen bekannt vor? Warum kommen Sie Ihnen bekannt vor? Würden Sie sie beim Kommunizieren in der deutschen Sprache häufig benutzen? Wenn ja, warum würden Sie sie beim Deutschsprechen benutzen? Begründen Sie Ihre Antwort ${ }^{16}$.

15 Bei dieser Aufgabe soll die kognitive Strategie zum Verständnis von zusammengesetzten Wörtern eingesetzt werden.

${ }^{16}$ Bei dieser Lernstrategie handelt es sich konkret um die Verwendung von schon aus der Muttersprache bekannten Internationalismen seitens der spanischsprachigen Lernenden. 
Aufgaben zur Ermittlung des Situationskontextniveaus und zur Einübung von Kommunikationsstrategien zur Planung der bevorstehenden Situation:

1. Informieren Sie sich über die in der bevorstehenden Kommunikationssituation zu verwendende Textsorte. Zur Einsetzung dieser Kommunikationsstrategie beantworten Sie folgende Fragen :

Um welche Situation handelt es sich im Text? Welche Wörter und sprachlichen Strukturen können in dieser Situation vorkommen? Kennen Sie sich mit dem in dieser Situation möglicherweise aufgerufenen Wortschatz und den Sprachstrukturen aus? Falls nicht, wie reagieren Sie? Müssen Sie sich dann auf diese Situation sprachlich vorbereiten? Wie würden Sie dies angehen?

Wer ist der Absender des Textes? Ist er eine einzelne Privatperson oder eine Firma? An wen wendet sich der Text? Richtet er sich an denselben Absender oder an andere Adressaten? Handelt es sich hierbei um einzelne oder mehrere Personen?

Wie ist der Interaktionsgrad der Kommunikation zwischen den beiden, Adressaten und Empfänger? Ist er hoch, moderat oder niedrig?

Wovon handelt grundsätzlich die durch die Website des gesundheitstouristischen Betriebs stattfindende Kommunikation?

Wer ist der Verkäufer? Wer ist der potenzielle Konsument der angebotenen Produkte?

2. Suchen Sie die Sprachmittel zum Ausdruck der Höflichkeit aus dem Text heraus.

Darf man im Deutschen die Kommunikationspartner duzen?

3. Kennen sich der Adressat und der Absender? Sind sie miteinander befreundet? Duzt der Absender des Textes seine möglichen Adressaten? Dürfen sie per Du kommunizieren? Müssen sie sich siezen? Welche soziopragmatischen Konventionen verwendet der Absender im Text?

4. Wie ist der Ort der Kommunikation? Privat oder öffentlich? 


\section{Ist die Domäne privat oder geschäftlich?}

6. Sind der Absender und der Empfänger im gleichen Raum oder befinden sie sich an unterschiedlichen Orten? Teilen sie dann den gleichen kommunikativen Ort?

7. Wie ist die zeitliche Interaktion durch die Website des gesundheitstouristischen Betriebs? Ist sie zeitgleich oder zeitversetzt? Kommunizieren sie also zur gleichen Zeit miteinander (wie z. B. bei einer persönlichen Konversation oder bei einem telefonischen Gespräch) oder muss jeder Kommunikationsteilnehmer eine gewisse Zeit warten, bis er jede Botschaft (die entsprechende E-Mail also) bekommt?

8. Wenn die Kommunikation durch die Website des gesundheitstouristischen Betriebs zeitversetzt stattfindet, haben Sie etwas Zeit zur Verfügung um mit dem Kunden zu kommunizieren. Währenddessen könnten Sie sich verschiedener Hilfsmittel bedienen, um die Anfragen der Kunden zu beantworten. Welche Hilfsmittel würden Sie dann heranziehen? Vielleicht benutzen Sie ein oder mehrere Hilfskommunikationsmittel, wie etwa die folgenden: Orthografische Computerkorrektoren, Vorlagen anderer, ähnlicher Texte, eigene Notizen, Grammatikbücher, das Lehrbuch, Wörterbücher, usw.

9. Ist der Kanal des Textes schriftlich oder mündlich?

10. Viele Texte enthalten Bilder. Stimmen die Informationen auf diesen Bildern mit den Informationen mancher Texte überein? Wenn ja, helfen diese Ihnen, den unbekannten Wortschatz zu erschließen?

11. Über welches Medium wird die erwähnte Textsorte vermittelt?

12. Ist der Text ein vergänglicher Text oder auf einem festen Format gespeichert? Kann man öfter auf ihn zugreifen? Lassen sich im Internet ähnliche Texte finden?

13. Ist die Website des gesundheitstouristischen Betriebs ein eigenständiger Text oder ist sie Teil eines anderen?

14. Was ist Threr Meinung nach das Ziel des Textes? Animiert er dazu, etwas zu machen? 
15. Welche Informationen soll der Text vermitteln?

16. Ist der Text unterhaltend? Versucht der Text, den Leser zu unterhalten?

17. In welchem sprachlichen Bereich findet die Kommunikation statt? Geht es dabei um eine Kommunikation unter Fachleuten? Ist es eine Konmunikation zwischen Fachleuten und Nichtfachleuten? Oder eher eine Kommunikation unter Laien?

18. Welchem Fachbereich würden Sie den Text zuordnen?

19. Basiert der Text auf reellen Begebenheiten oder auf fiktiven Geschichten?

20. Wenn die Website des gesundheitstouristischen Betriebs fiktive Geschichten oder Informationen enthält, wozu sollen sie dann dienen?

Aufgaben zur Ermittlung des Situationskontextniveaus und zur Einübung von Kommunikationsstrategien zur Produktion von fremdsprachigen Texten:

Wichtiger Hinweis: Bei der Erledigung dieser Aufgaben können Sie übrigens folgende Kommunikationsstrategien verwenden: Handbücher über deutsche Geschäftskorrespondenz heranziehen; echte Geschäftsbriefe und E-Mails benutzen; einen Computerübersetzer heranziehen; im Wörterbuch oder in der selbst angelegten Wortschatzliste, in dem in der schriftlichen Geschäftssituation produzierten Wortschatz nachschlagen; eine Karteikarte mit Schlüsselwörtern konsultieren, wenn Probleme im Kommunikationsprozess auftreten; auf eine selbst angelegte Liste mit deutschen Sätzen und deren spanischen Äquivalenzen zurückgreifen, wenn Probleme im Kommunikationsprozess auftreten.

1. Beantworten Sie folgende Anfragen:

1. Anfrage:

Von: markuswilms456@deut.de

Betreff: Reservierungsbestätigung

Sehr geehrte Damen und Herren, 
Ich möchte gerne vom 20. März bis zum 05. April ein Doppelzimmer buchen.

Außerdem würde ich gerne wissen, ob in Threm Wellnesshotel auch sehnenentzündungstherapien durchgeführt werden. Ich würde gerne weitere Informationen zu den Therapiearten von Sehnenentzündung bei Thnen bekommen.

Mit freundlichen Grüßen,

[Platz für die eigenhändige Unterschrift]

Markus Wilms

*(Sehnenentzündung bedeutet "tendinitis" auf Spanisch)

2. Anfrage:

Von: tanjamauer@deut.de

Betreff: Auskunft über Hier fehlt ein Wort

Sehr geehrte Damen und Herren,

Mein Mann und ich haben vor, eine Woche in Ihrem Wellnesshotel $z u$ verbringen. Ich wäre Ihnen dankbar, wenn Sie mir Auskunft über die Preise für ein Doppelzimmer im Zeitraum zwischen dem 16.05 . und dem 22.06.2014 geben.

Außerdem würde ich gerne wissen, ob in Threm Wellnesshotel auch Sehnenentzündungstherapien durchgeführt werden.

Welche Therapiearten von Sehnenentzündung bekommt man bei Ihnen?

Ich bekomme 60 Prozent der Behandlung von der Allgemeinen Krankenkasse finanziert. Haben Sie auch eine Vereinbarung mit der Allgemeinen Krankenkasse?

Mit freundlichen Grüßen,

[Platz für die eigenhändige Unterschrift] Tanja Mauer

2. Versuchen Sie, in Gruppenarbeit eine Website von ungefähr zwei bis drei Seiten, zu erstellen. Wie würden Sie dabei verfahren? 
Würden Sie dabei kurze oder längere Sätze benutzen? Was würden Sie machen, damit Thre Website bei einem möglichst weitreichenden Publikum ankommt? Würden Sie sie auch mit Bildern schmücken? Würden Sie die soziopragmatischen Konventionen beachten?

3. Suchen Sie im Netz nach einem Wellnesshotel in Deutschland und schreiben Sie eine E-Mail mit den folgenden Informationen: Entfernung des Hotels vom Stadtzentrum; Preis eines Doppelzinmers; Preis folgender Wellnessdienstleistungen: AntiStress-Behandlungen, Kopfmassagen, Gesichtsmassagen, Rückenmassagen; Day SPA-Angebote, Zimmerreservierung: vom ... bis. Beachten Sie dabei die Forschungsergebnisse der Applikation der Theorie des Registers.

4. Schreiben Sie eine Reservierung mit den folgenden Informationen: Entfernung des Hotels vom Stadtzentrum; Preis eines Doppelzinmers; Preis einer Anti-Stress-Behandlung, Zeitraum. Beachten Sie dabei die Forschungsergebnisse der Applikation der Theorie des Registers.

5. Geben Sie Bestellungen für Wellnessdienstleistungen auf und bestätigen Sie sie auch. Beachten Sie dabei die Forschungsergebnisse der Applikation der Theorie des Registers.

6. Klicken Sie sich durch Webseiten von Wellnesshotels und informieren Sie sich über die Therapien gegen folgende Beschwerden: Gelenkentzündung, Atembeschwerden, Schulterschmerzen, Sehnenentzündung, Rückenschmerzen, Hüftschmerzen, Knieschmerzen.

7. Suchen Sie in Websites von Wellnesshotels nach passenden Therapien gegen Lumbalgie und denken Sie sich eine Fortsetzung für den nachfolgenden Dialog aus:

Physiotherapeutin: Leiden Sie an Rückenschmerzen?

Patient: Ja.

Physiotherapeutin: Was für Rückenschmerzen haben Sie?

Patient: Ich weiß es nicht ganz genau.

Futhark 9 (2014)

ISSN $1886-9300$

Hernández, Die Website, 153-202 
Physiotherapeutin: Tut es Ihnen hier weh oder weiter unten?

Patient: Es tut mir ein bisschen weiter unten weh.

Physiotherapeutin: Das ist die Lendengegend. Dann würde ich Ihnen folgende Massagen empfehlen...

8. Rollenspiel. Erstellen Sie eine Besichtigung durch ein Wellnesshotel. Dabei sollten Sie Ihre Gäste über die Einrichtungen, Räume, Dienstleistungen, usw. informieren:

Rezeptionist: Vielleicht könnten wir jetzt eine Besichtigung durch das Hotel machen. Hier ist also der/die/das.... Der/die/das... sind hier geradeaus. Wir gehen jetzt die Treppe runter. Das ist das... Jetzt nehmen wir den Aufzug. Hier rechts ist... Dort sehen Sie... Hier ist auch... Die Rezeption haben Sie schon gesehen. Wir gehen nach links. Da sehen Sie... Da geradeaus ist... . Wir gehen nach rechts. Da ist... Jetzt gehen wir zurück. Das ist wieder... Dann sehen sie rechts... Rechts ist der Arzt... Links ist die Physiotherapeutin ...

Zur Erledigung dieser Sprachaktivität können Sie Websites von Wellnessbetrieben oder Wellnesswörterbücher heranziehen.

9. Ihre deutschen Kunden möchten gerne Körpermassagen über diese Webseite buchen: www.bad-hotel.de. Erklären Sie ihnen auf Deutsch, welche Körpermassagen man auf dieser Webseite buchen kann.

10. Sie sind Angestellter eines Wellness-Hotels (www.badhotel.de). Ein deutscher Kunde fragt Sie nach den folgenden Informationen: Preis für ein Zimmer pro Nacht, Fittnessstudio und Preis für 4 Wellnessdienstleistungen. Gehen Sie bitte auf die Webseite des Hotels und suchen Sie nach den verlangten Informationen. Geben Sie die Informationen auf dieser Webseite mündlich an den Kunden weiter.

11. Erklären Sie Ihren spanischen Mitstudenten die folgenden Begriffe auf Spanisch: Magnetfeldtherapie, Maniküre, Osmanisches Dampfbad, Reduktionsdiät, Rosenblütenbad.

Erklären Sie Ihren spanischen Mitstudenten die folgenden Begriffe auf Deutsch: Sanarium, Salzölbad, Schwebebad, Spinning, Sudarium. 
12. Übersetzen Sie die nachfolgende E-Mail ins Deutsche:

\begin{tabular}{|l|c|}
\hline Absender: & Emst Siebelds esiebelds@firmade.de \\
\hline Empfänger: & $\begin{array}{c}\text { Wellness-Hotel zur Laterne } \\
\text { Laterne@wellnesshotelskette.de }\end{array}$ \\
\hline Datum: & $03.05 .201316: 36$ \\
\hline Anlagen: & \\
\hline Betreff: & Más preguntas \\
\hline
\end{tabular}

Estimados señoras y señores,

Tengo una pregunta. Nos gustaría informarnos sobre los tratamientos para la espalda.

Un cordial saludo, Ernst Siebelds

13. Sie sind Angestellter eines Wellness-Hotels. Ein deutscher Kunde möchte bei Thnen Körperbehandlungen buchen. Er möchte sich folgende Informationen einholen: Therapien gegen Rückenschmerzen; Preis für ein Doppelzimmer; Wellnesseinrichtungen (Sauna, Dampfbad, Infrarotkabine, Erlebnisdusche, usw.). Gehen Sie bitte auf die erwähnte Webseite und suchen Sie nach den verlangten Informationen. Geben Sie dem Kunden diese Informationen auf dieser Webseite schriftlich wieder. Versuchen Sie wenn möglich, eine E-Mail mit diesen Informationen zu verfassen. Zur Erledigung dieser sprachmittlerischen Aktivität können Sie Websites von Wellnessbetrieben oder Wellnesswörterbücher heranziehen.

\section{SCHLUSSFOLGERUNG}

Nach Beendigung dieser Forschung lässt sich $u$. E. die nach wie vor gegenwärtige Relevanz der vor mehr als vierzig Jahren entstandenen Theorie des Registers als Forschungsrahmen im berufs- 
und fachbezogenen Fremdsprachenunterrichts bestätigen. Dieses didaktische Analyseverfahren hat uns eine Reihe von Analyseparametern für den Einsatz von realen fachbezogenen Texten in unserem tourismusbezogenen Deutschunterricht verschafft, die uns, wie schon im Titel dieses Artikels erwähnt, die folgende Aktion ermöglicht hat: die grundlegenden Sprach- und Situationskontextmerkmale des authentischen Textes die Website des gesundheitstouristischen Betriebs didaktisch aufzubereiten.

Jene Parameter haben uns ermöglicht, den gerade erwähnten sprach- und kontextbezogenen Stoff $\mathrm{zu}$ bestimmen, sowie das Lehrmaterial für unseren berufs- und fachbezogenen Deutschunterricht $\mathrm{zu}$ bearbeiten. Anhand der sprachbezogenen Parameter haben wir die häufigsten sprachlichen Themen in den Ausgangstexten expliziert: allgemeiner Wortschatz und semitechnischer Wortschatz aus der Tourismus- und der Wellnesssprache; die Wortbildungsressourcen Nominalkomposition, Komposition aus Substantiv und Adjektiv (Partizip), Komposition mit Bindestrich, Komposition aus deutschem und Fremdwort, Ableitung mit Suffixen, Ableitung durch Halbpräfixe und Übernahme von Fremdwörter; die Zeitform Indikativ Präsens; die Personalformen in der dritten Person Singular; die Höflichkeitsform; der Indikativ- und der Imperativmodus; Aktiv- und Passivsätze; einfache Sätze; Relativsätze; Infinitivsätze; und kurze und lange Satzglieder (sogar Satzglieder mit mehr als drei Spracheinheiten).

Durch die Applikation der kontextuellen Parameter dieses Untersuchungsverfahrensmodells ließen sich die sozialen, pragmatischen, funktionellen und situationsbedingten Hauptfaktoren der Website des gesundheitstouristischen Betriebs identifizieren: pluralischer und betrieblicher Absender; gerichtet an andere Rezipienten; Rezipient pluralischer Natur; hierarchische Beziehung zwischen "Chef" und "Untergebenem"; moderate Interaktion; Beziehungen von gegenseitigem Respekt; öffentlicher Kommunikationsraum; geschäftliche Domäne; ungeteilter Kommunikationsraum; zeitversetzte Kommunikation; schriftlicher Kommunikationsmodus; Kombination von schriftlichen und ikonischen Zeichen; permanenter Text; elektronisches permanentes 
Obertragungsmittel; veröffentlicht; unabhängiger oder Bestandteil einer größeren Spracheinheit; Überzeugungs- und Verkaufsziele; hoher Informationsvermittlungsgrad; unterhaltend; keine Belehrung; kein Ausdruck persönlicher Gefühle; keine Verbesserung der interpersönlichen Beziehungen; keine Äußerung von Haltungen; positive Meinungen über die angebotenen Produkte und Dienstleistungen; allgemeinsprachlicher und halbspezialisierter Wortschatz; moderater fachlicher Sprachgrad; touristisches Gesundheits-Wellnessdienstleistungsfeld; wirklich beschriebene Ereignisse/Gegebenheiten.

Ebenso hat uns die Theorie des Registers als Grundlage für die Ausarbeitung von Aufgaben pragmatisch-kontextueller Natur zur Vermittlung des ermittelten Lernstoffs gedient, bei denen die Lemenden auRerdem Sprachlernstrategien einüben und direkten Kontakt zu authentischen deutschsprachigen Texten aufnehmen sollen.

Auf Basis von deren morphologischen Parametern ließen sich Aufgaben zur Praxis folgender direkten Sprachlernstrategien entwickeln: eine Memorierungsstrategie zur Aufarbeitung von mehreren Wörtern mit dem gleichen Grundmorphen ${ }^{17}$, eine Memorierungsstrategie zum Erwerb von komplexen Wörtern ${ }^{18}$, kognitive Strategien zur Folgerung von Grammatikphänomenen ${ }^{19}$, kognitive Strategien zur Vereinfachung der Lektüre von Texten mit langen zusammengesetzten Wörtern ${ }^{20}$ und eine Kompensationsstrategie zur Vereinfachung der Produktion von fremdsprachigen Texten ${ }^{21}$.

\footnotetext{
17 Siehe Aufgaben 1 und 2 zum Erwerb von Sprachelementen und zur Einsetzung von Memorierungsstrategien.

${ }^{18}$ Siehe Aufgabe 3 zum Erwerb von Sprachelementen und zur Einsetzung von Memorierungsstrategien

${ }^{19}$ Siehe Aufgaben 1 bis 5 zum Erwerb von Sprachelementen und zur Einsetzung von kognitiven Lemstrategien zur Schlussfolgerung von Grammatikphänomenen.

${ }^{20}$ Siehe Aufgabe 6 zum Erwerb von Sprachelementen und zur Einsetzung von kognitiven Lernstrategien zur Erleichterung des Lesens in der Fremdsprache.

21 Siehe Aufgabe 1 zum Erwerb von Sprachelementen und zur Einsetzung von Kompensationsstrategien.
} 
Mithilfe von deren lexikalischen Parametern wurde eine Memorierungsstrategie zur Aufarbeitung von Fachwortschatz, halbfachspezifischem Wortschatz und allgemeinem Wortschatz gebildet ${ }^{22}$.

Unter Heranziehung der darauf basierenden Syntaxanalyse haben wir folgende kognitive Strategien entwickelt: eine Strategie zur Folgerung von Grammatikphänomenen ${ }^{23}$, eine Strategie zur Vereinfachung der Lekture von Texten mit langen Sätzen ${ }^{24}$ und eine Strategie zur Vereinfachung der Lektüre von Texten mit langen Satzgliedern ${ }^{25}$.

Auf Grundlage von deren kontextuellen Analysekriterien haben wir folgende Kommunikationsstrategien entworfen: Strategien zur Planung der bevorstehenden Kommunikationssituation in der Fremdsprache ${ }^{26}$ und Strategien zur Produktion von fremdsprachigen Texten $^{27}$.

Zuletzt sei erwähnt, dass sich unter Anwendung der Theorie des Registers einige relevante Informationen zur Herstellung von deutschsprachigen, tourismusbezogenen schriftlichen Werbetexten identifizieren lassen: leicht verständliche Sprache; zahlreiche Fotos; große Anzahl an langen Satzgliedern und zusammengesetzten Wörtern; zeitversetzte Kommunikation; reich an Fremdwörtern; Einhaltung der soziopragmatischen Konventionen; adressiert an das möglichst große Publikum; umfangreicher halbspezialisierter Wortschatz aus dem Tourismusbereich; moderater fachlicher Sprachgrad; geschäftliche Natur; Ziel: Verkauf von Produkten und

22 Siehe Aufgaben 4 bis 9 zum Erwerb von Sprachelementen und zur Einsetzung von Memorierungsstrategien.

${ }_{23}$ Siehe Aufgaben 7 bis 16 zum Erwerb von Sprachelementen und zur Einsetzung von kognitiven Lemstrategien zur Schlussfolgerung von Grammatikphänomenen. 24 Siehe Aufgabe 1 zum Erwerb von Sprachelementen und zur Einsetzung von kognitiven Lernstrategien zur Erleichterung des Lesens in der Fremdsprache ${ }_{25}$ Siehe Aufgaben 2 bis 6 zum Erwerb von Sprachelementen und zur Einsetzung von kognitiven Lemstrategien zur Erleichterung des Lesens in der Fremdsprache. ${ }^{26}$ Aufgaben zur Ermittlung des Situationskontextniveaus und zur Einübung von Kommunikationsstrategien zur Planung der bevorstehenden Situation ${ }^{27}$ Aufgaben zur Ermittlung des Situationskontextriveaus und zur Einübung von Kommunikationsstrategien zur Produktion von fremdsprachigen Texten. 
Dienstleistungen; Vermittlung von positiven Meinungen aber die angebotenen Produkten und Dienstleistungen; usw.

\section{BIBLIOGRAPHIE}

Alcaraz Varo, Enrique, El Inglés profesional y académico, Madrid, Alianza Editorial, 2000.

Bässiter, Roland, Qualitätsnioemus und Gesundheitskompetenz im österreichischen Kur- und Wellnes-Tourismus, Wien, Institut für Sportwissenschaft der Universităt Wien, 2003.

DE BEAUGRANDE, Robert, „Register in Discourse Studies: A Concept in Search of a Theory", in Ghadessy, Mohsen (Eds.), Register Analysis: Theory and Practic, London, Pinter, 1993, S. 7-25.

BiBER, Douglas, „An Analytical Framework for Register Studies", in BIBER, Douglas; FNEGAN, Edward (Eds.), Sociolinguistic Perspectives on Register, New York, Oxford University Press, 1994, S. 31 - 56.

Bosch, Ramon et alli, Turismo y Medio Ambiente, Madrid, Editorial Universitaria Ramón Areces, 1998.

CORBACHO SÁNCHEZ, Alfonso, "Die metaphorische Konzeptualisierung der Wirtschaft in touristischen Texten. Deutsch und Spanisch im Kontrast", in Muttersprache, 2014, Nr. 124 (1), S. 26-40.

Dudley-Evans, T.; ST. JoHN, M. J.: Deoelopments in English for Specific Purposes, Cambridge University Press, Cambridge, 1998.

European Route of thermal heritage and thermal towns, Acqui Terme: European Historical Thermal Town Association, 2010, S. 8. URL: http://termalismo.ourense.es/wpcontent/uploads/2013/01/EHTTA_european_route.pdf. Letzter Zugriff: 09.11. 2013.

EWER, J. R; LATORRE, G., A Course in Basic Scientific English, London, Longman Publishing Group, 1969.

GarCíA MAYO, M. P., English for Specific Purposes: Discourse Analysis and Course Design, Bilbao, Universidad del País Vasco, 2000.

GlabONIAT, M. et al., Profile Deutsch, Langenscheidt, München, 2002.

Glabonat, M. et al., Profile Deutsch A - C, München, Langenscheidt, 2005.

HallidaY, M. A. K., Language as a Social Semiotic, London, Edward Arnold, 1978.

- et al, The linguistic sciences and language teaching, London, Longman, 1964. HUTCHINSON, T.; WATERS, A., English for Specific Purposes. A learning-centred approach, New York, Cambridge University Press, 1987. 
Innovativer Gesundheitstourismus in Deutschland, Berlin, Bundesministerium für Wirtschaft und Technologie, 2011. URL: http://www.innovativergesundheitstourismus.de

/ fileadmin/ user_upload/pdf/Leitfaden_Gesundheitstourismus. pdf. Letzter Zugriff: 13.11. 2013.

JORDAN, R. R., English for Academic Purposes: A guide and resource book for teachers, Cambridge, Cambridge University Press, 1997.

JUSZCZAK, J., Medizintourismusdestination Deutschland, Untersuchungsreihe des Fachbereichs Wirtschaftswissenschaften zum Markt des Medizintourismus, Sankt Augustin, Hochschule Bonn-Rhein-Sieg, 2012.

LOPEZ MORALES, Manuel, "El potencial turístico de los balnearios", in Estudios Turísticos, 2003, Nr. 157, S.125-145.

OXFORD, Rebecca L., Language learning strategies: what every teacher should know, Boston, Heinle \& Heinle Publishers, 1990.

Ökonomisches Schwergewicht: Wir sind Gesundheitstourismus! (Pressemitteilung, 31.05.2013), in URL: http://www.deutscherheilbaederverband.de/Oekonomisches-Schwergewicht-Wir-sindGesundheitstourismus-707904. html. Letzter Zugriff: 13.11. 2013.

PÉREZ RUIZ, Leonor, Análisis retórico contrastivo: el resumen lingüístico y médico en inglés y español (Tesis doctoral), Universidad de Valladolid, 2001.

Plan General de Turismo Sostenible de Andalucia 2008 - 2011, Sevilla, Consejería de Turismo, Comercio y Deporte de la Junta de Andalucía, 2008.

„Real Decreto 1143/2006". URL: http://www.boe.es/boe/dias/2006/10/20/pdfs/ A36639-36641.pdf. Letzter Zugriff: 09.11. 2013.

ROBINS, R.H, "The contribution of John Rupert Firth to linguistics in the first fifty years of Lingua", in Lingua, Nr. 100, 1997, S. 205 - 222.

RUCCI, Marco, "Working with register in the classroom: the spanish case”, in The Interpreters' Newsletter, 1999, Nr. 9, S. 191-98.

SCHROEDER, G., Lexikon der Tourismuswirtschaft, Hamburg, Hannelore Niedecken, 2002.

SUAU JIMÉNEZ, Francisca, „El género y el registro en la traducción del discurso profesional: un enfoque funcional aplicable a cualquier lengua de especialidad especialidad", in El ELE para contextos profesionales (Cultura e intercultura en la enseñanza del español como lengua extranjera), 2001, URL: http://www.ub.es/filhis/culturele/tinasuau.html. Letzter Zugriff: 27.09.2013.

TRIM, J. et al., Gemeinsamer europäischer Referenzrahmen für Sprachen: lernen, lehren, beurteilen, Berlín, Langenscheidt, 2001.

VOGEler RUIZ, Carlos; HeRnÁNDEZ ARMAND, Enrique, El mercado turístico: estructura, operaciones y procesos de producción, Madrid, Editorial Centro de Estudios Ramón Areces, 1995. 
ZEOUAN, Liu, "Register Analysis as a Tool for Transla ment", in Translation Jounal, $\mathrm{Nr}$. http://www.bokorlang.com/journal/25register.htm. 16.11.2013.

\section{Onlinewörterbücher}

http://www.wellness-lexikon.de/begriffe.htm,

http:// www.wellnessverband.de/infodienste/lexikon/listview.php/

\section{Textkorpus}

hitp://www:georgshoehe.de

http:// www oceano.de

http:/ / www:panorama-hotel.de

http://nww.schoennh-seefeld.com

http://nww:strandhotel-kurhaus-juist.com

http://www.schwarzat

http://www.wartherhof.at

http://www.jammertal.de

http://www.thermenbussloo.nl/de

http://www.westingrandmunichcom

http://www.zentral.it/

http://www.zugbruecke.de/ 